\title{
Identification of Glycoproteins from Mouse Skin Tumors and Plasma
}

\author{
Yuan Tian • Karen S. Kelly-Spratt • \\ Christopher J. Kemp • Hui Zhang
}

Published online: 4 September 2008

(C) Humana Press 2008

\begin{abstract}
Introduction Plasma has been the focus of testing different proteomic technologies for the identification of biomarkers due to its ready accessibility. However, it is not clear if direct proteomic analysis of plasma can be used to discover new marker proteins from tumors that are associated with tumor progression. In this paper, we reported that such proteins can be detected in plasma in a chemical-induced skin cancer model in mice.

Materials and Methods We analyzed glycoproteins from both benign papillomas and malignant carcinomas from mice using our recently developed platform, solid-phase extraction of glycopeptides and mass spectrometry, and identified 463 unique N-linked glycosites from 318 unique glycoproteins. These include most known extracellular proteins that have been reported to play roles in skin cancer development such as thrombospondin, cathepsins, epidermal growth factor receptor, cell adhesion molecules, cadherins, integrins, tuberin, fibulin, and TGF $\beta$ receptor. We further investigated whether these tumor proteins could be detected in plasma from tumorbearing mice using isotope labeling and 2D liquid chromatography/matrix-assisted laser desorption/ionization tandem mass spectrometry.
\end{abstract}

\section{Y. Tian}

Department of Pathology, Johns Hopkins University,

Baltimore, MD 21231, USA

K. S. Kelly-Spratt • C. J. Kemp

Human Biology and Public Health Science,

Fred Hutchinson Cancer Research Center,

Seattle, WA 98109, USA

\section{H. Zhang $(\bowtie)$}

Department of Pathology, Johns Hopkins University, 1550 Orleans Street, CRBII, Room 3M-03,

Baltimore, MD 21231, USA

e-mail: hzhang32@jhmi.edu
Results and Discussion Two tumor glycoproteins, Tenascin-C and Arylsulfatase B, were identified and quantified successfully in plasma from tumor bearing mice. This result indicates that analysis of tumor-associated proteins in tumors and plasma by a method using glycopeptide capture, isotopic labeling, and mass spectrometry can be used as a discovery tool to identify candidate tumor proteins that may be detected in plasma.

Keywords Cancer. Plasma - Tumor - Glycoprotein . Proteomics · Mass spectrometry · MALDI-TOF/TOF. MS/MS $\cdot$ Serum $\cdot$ Plasma $\cdot$ Biomarker

\section{Introduction}

Despite the great increase in understanding of cancer at the molecular level, cancer remains as the second most common cause of death in the USA. Survival rates for many common cancer types have changed little over the past two decades [1]. If cancer is detected early, prior to metastatic spread, survival rates are vastly improved [1]. For this reason, improvements in the ability to detect cancer early may significantly reduce mortality from cancer. Plasma has been the focus for applying different proteomic technologies for the identification of biomarkers for early detection due to its ready accessibility. These developments include depletion of the most abundant plasma proteins [2] and extensive fractionation of proteins or peptides prior to mass spectrometric analysis [3-5]. However, proteins discovered by serum profiling are often well-known, highabundance, classical serum proteins [6], not likely to be specifically derived from cancer tissue. Useful biomarkers for cancer detection in blood are those proteins released specifically from cancer tissues (overexpression of cancer 
proteins), indicators of a specific response of the host to cancer cells, or leaking of organ restricted proteins to blood due to structural changes in the microenvironment surrounding cancer cells (leaking of normal proteins such as PSA) [7]. Tumor proteins that are detectable in both benign and malignant tumors, as well as plasma can serve as candidate proteins for early detection of cancer. Detection of these proteins in plasma is critical to evaluate proteomic technologies for the biomarker discovery.

In an attempt to identify the proteins derived from cancerous tissue that are most likely to be present in blood, we employed our recently developed glycoproteomic analysis method using solid-phase extraction of N-linked glycopeptides (SPEG) [8-10]. The method has several advantages. First, most cell-surface and secreted proteins are glycosylated, and disease-associated glycoproteins (secreted by cells, shed from their surface, or otherwise released) are likely to enter the bloodstream and thus represent a rich source of potential disease markers [11]. Second, the reduction in complexity achieved by focusing on the glycoprotein subproteome in both tissues and plasma translates into favorable limits of detection, thus increasing the likelihood that the same polypeptide will be detectable in both tissue and serum [8, 12, 13]. Third, aberrant glycosylation is a fundamental characteristic of oncogenesis and tumor progression [14], and this method allows us to identify proteins changed in glycosylation but not necessarily changed in total protein abundance. Finally, specific mass-spectrometry-based methods and affinity reagents can be developed for the specific and sensitive detection of identified tissue proteins in plasma [15], selective isolation of specific proteins or peptides using affinity reagents [16], or the recently developed targeted approach using multiple reaction monitoring (MRM) [17-19].

The chemically induced two-stage mouse skin carcinogenesis model has been used for decades to study the genetic, molecular, and biologic basis of tumor development [20]. For example, the concepts of tumor initiation and promotion were derived from this model. In this model, the backs of 8-week-old mice treated with the carcinogen 7,12-dimethylben[a] anthracene (DMBA) followed by multiple treatments with the tumor promoter 12-o-tetradecanoylphorbol-13-acetate (TPA). Benign tumors (papillomas) develop after 8 weeks, and a small percentage of these progresses to malignant invasive carcinomas after a long latency [20]. The ability to quantify both benign and malignant tumor growth permits analysis of genes and environmental factors that affect tumor progression. More recently, the two-stage skin tumor model has been used to improve proteomic technologies for biomarker discovery using serum protein profiling [12]. We have identified several serum proteins for which the abundance is increased in correlation with the chemical induction of skin cancer in mice. However, these proteins are likely not markers for the specific diagnosis of skin cancer. A major advantage of this mouse skin carcinogenesis model is that plasma samples can be taken from mice before and after tumor development. As both benign and malignant tumors and plasma samples can be obtained from the same mice, this facilitates analysis of protein changes in plasma associated with tumor development.

In this paper, we report a two-step strategy for detection of tumor-associated proteins in plasma: the first step was to analyze extracellular proteins from normal skin, papillomas, and carcinomas and identify tumor-associated proteins; the second step was to detect the tumor-associated proteins in plasma using a tissue-targeted approach and isotope labeling [7]. Using our recently developed method of SPEG and mass spectrometry [8-10], we analyzed matched benign and cancerous tumors from four tumor-bearing mice as well as normal skin tissues from four control mice, and identified 463 unique N-linked glycosites from 318 glycoproteins. More than 40 identified glycoproteins were elevated in carcinomas. Two of the tumor-associated proteins, Tenascin-C and Arylsulfatase B, were further detected and quantified in plasma from the same cancerbearing mice using isotope labeling and 2D liquid chromatography/matrix-assisted laser desorption/ionization tandem mass spectrometry (LC-MALDI-MS/MS). This result indicates that direct proteomic analysis of tumors and plasma using glycopeptide capture, isotopic labeling, and mass spectrometry can be used to discover new cancer-derived proteins in plasma.

\section{Method and Materials}

\section{Materials}

Hydrazide resin and sodium periodate were from Bio-Rad (Hercules, CA, USA); PNGase F was from New England Biolabs (Ipswich, MA, USA); sequencing grade trypsin was purchased from Promega (Madison, WI, USA); C18 columns were from Waters (Milford, MA, USA); $\alpha$-cyano4-hydroxycinnamic acid (CHCA) was from Agilent (Palo Alto, CA, USA); iTRAQ reagent and mass calibration standards were purchased from Applied Biosystems (Foster City, CA, USA). All other chemicals were purchased from Sigma-Aldrich (St. Louis, MO, USA).

Tissues and Plasma from Chemical-Induced Mouse Skin Tumors

Skin tumors were induced in four NIH01a mice using the DMBA/TPA two-step protocol. A single dose of DMBA (Sigma; $25 \mathrm{mg}$ in $200 \mathrm{ml}$ of acetone) was applied to the shaved 
backs of four 8-week-old mice. Initiated treated skin was promoted with TPA twice a week for 15 weeks. This gave rise to papillomas that were hyperplastic, well-differentiated, benign lesions consisting of keratinocytes together with stroma tissue. Papillomas appeared as early as 8 weeks after the first treatment of DMBA and continued to grow for the next several months. A small percentage of these benign papillomas $(\sim 20 \%)$ progressed to squamous cell carcinomas. All the mice were killed when carcinomas appeared in all four treated mice. Four littermate mice were left untreated for normal skin tissues. Papillomas and carcinomas, as well as normal skin from untreated mice were snap-frozen in liquid nitrogen. Retro-orbital bleeds were collected from each treated mouse before chemical treatment and after development of chemical-induced carcinomas. The only difference between the normal and cancer tissues is the chemical-induced cancer. Whole blood $(0.25 \mathrm{ml})$ was collected from the retro-orbital sinus into a long (9 in.) sterile glass Pasteur pipet. The whole blood was placed in a K3EDTA-coated $1.5 \mathrm{ml}$ microcentrifuge tube and centrifuged at $4{ }^{\circ} \mathrm{C}$ for $5 \mathrm{~min}$ at $3,000 \mathrm{rpm}$. Plasma was collected, carefully avoiding cellular contamination. All tumor tissues and plasma were placed in cryovials and frozen in liquid nitrogen.

\section{Peptide Extraction from Skin Tumor Tissues}

Frozen tumor tissues (100 mg each) were sliced into 1 - to $3-\mathrm{mm}^{3}$ thickness and incubated in $200 \mu \mathrm{l}$ of $5 \mathrm{mM}$ phosphate buffer and vortexed for 2-3 $\mathrm{min}$. Then, the samples were sonicated for $5 \mathrm{~min}$ in an ice-water bath. Trifluoroethanol (TFE, 200 $\mu$ l) was added to the sample and incubated at $60^{\circ} \mathrm{C}$ for $2 \mathrm{~h}$ followed by sonication for $2 \mathrm{~min}$. Protein disulfide bonds were reduced by $5 \mathrm{mM}$ tributylphosphine with $30-\mathrm{min}$ incubation at $60^{\circ} \mathrm{C}$. Iodoacetamide (10 $\mathrm{mM})$ was applied to the mixture and incubated in the dark at room temperature for another $30 \mathrm{~min}$. The samples were diluted fivefold with $50 \mathrm{mM} \mathrm{NH}_{4} \mathrm{HCO}_{3}(\mathrm{pH}$ 7.8) to reduce the TFE concentration to $10 \%$ prior to the addition of trypsin at a ratio of 1:50 (w/w, enzyme/protein). Samples were digested at $37^{\circ} \mathrm{C}$ overnight with gentle shaking. The precipitate was discarded by centrifuge. Silver staining was used to test the effect of tryptic digestion. Four milligrams of total peptides from each sample was extracted from each tissue. Two milligrams of total peptide was used to extract $\mathrm{N}$-linked glycopeptides, according to the following steps.

\section{Peptide Extraction from Plasma}

Plasma $(20 \mu \mathrm{l})$ was added to $90 \mu \mathrm{l} 8 \mathrm{M}$ urea in $0.4 \mathrm{M}$ $\mathrm{NH}_{4} \mathrm{HCO}_{3}, 0.1 \%(w / v)$ sodium dodecyl sulfate (SDS) solution ( $\mathrm{pH} \mathrm{8.3)}$ and $10 \mu \mathrm{l} 120 \mathrm{mM}$ tris(2-carboxyethyl) phosphine in $\mathrm{dH}_{2} \mathrm{O}$ freshly prepared and incubated at $60^{\circ} \mathrm{C}$ for $1 \mathrm{~h}$. Proteins were alkylated by adding $10 \mu \mathrm{l} 160 \mathrm{mM}$ iodoacetamide and incubated at room temperature in the dark with shaking for another $30 \mathrm{~min}$. Samples were diluted by trypsin digestion buffer $\left(100 \mathrm{mM} \mathrm{NH}_{4} \mathrm{HCO}_{3}, \mathrm{pH} 8.3\right)$ to make the concentration of urea less than $2 \mathrm{M}$. Forty microliters of trypsin $(0.5 \mu \mathrm{g} / \mu \mathrm{l})$ was added to digest protein at $37^{\circ} \mathrm{C}$ overnight. SDS-polyacrylamide gel electrophoresis (SDS-PAGE) and silver staining were employed to check whether trypsin digestion was complete.

\section{Glycopeptide Capture from Tissue or Plasma}

N-glycopeptides were isolated from peptides using SPEG [9]. The enriched N-linked glycopeptides were concentrated by $\mathrm{C} 18$ columns and dried down and resuspended in $40 \mu \mathrm{l}$ $0.4 \%$ acetic acid prior to MS analysis.

Isotope Labeling of Peptides

The amount of glycopeptide was determined by bicinchoninic acid assay (Bio-Rad) prior to isotope labeling. Glycopeptides $(1 \mu \mathrm{g})$ from plasma of the retro-orbital bleeds before and after chemical-induced cancer and tumor tissues were dried and resuspended in $20 \mu \mathrm{l}$ of $50 \%$ dimethylformamide, $40 \% \mathrm{H}_{2} \mathrm{O}, 10 \%$ pyridine. Five microliters $10 \mathrm{mg} / \mathrm{ml} \mathrm{d} 0{ }^{13} \mathrm{C} 0, \mathrm{~d} 4{ }^{13} \mathrm{C} 0$, and $\mathrm{d} 4{ }^{13} \mathrm{C} 4$ succinic anhydride solution was added to glycopeptide samples and reacted at room temperature for $1-2 \mathrm{~h}$, then followed by C18 clean up to remove access succinic anhydride [8].

\section{Mass Spectrometry Analysis}

The peptides and proteins were identified using MS/MS analysis using an LTQ ion trap mass spectrometer (Thermo Finnigan, San Jose, CA, USA). Glycopeptides (1 $\mu \mathrm{g})$ were injected into a peptide cartridge packed with $\mathrm{C} 18$ resin and then passed through a $10 \mathrm{~cm} \times 75 \mu \mathrm{m}$ i.d. microcapillary high-performance liquid chromatography ( $\mu \mathrm{LC})$ column packed with $\mathrm{C} 18$ resin. The effluent from the $\mu \mathrm{LC}$ column entered an electrospray ionization source in which peptides were ionized and passed directly into the mass spectrometer. A linear gradient of acetonitrile from $5 \%$ to $32 \%$ over $100 \mathrm{~min}$ at flow rate of $\sim 300 \mathrm{nl} / \mathrm{min}$ was applied. During the LC-MS mode, data were acquired between $\mathrm{m} / \mathrm{z}$ of 400 and 2,000. Each sample was analyzed three times to increase the number of spectra used for spectral count.

Succinic-anhydride-labeled peptide $(5 \mu \mathrm{g})$ was analyzed by 2D Nano LC (Eksigent, Dublin, CA, USA) and MALDI-tandem time of flight (MALDI-TOF/TOF; Applied Biosystems). Briefly, online integration of $15-\mathrm{cm}-$ long $300 \mu \mathrm{m}$ strong cation exchange column (SCX) with 15-cm-long $300 \mu \mathrm{m}$ of C18-reverse phase liquid chromatograph (RPLC) was employed. Four SCX fractions of 0, 5, 50 , and $500 \mathrm{mM} \mathrm{KCl}$ and $3-45 \%$ linear acetonitrile 
gradient (containing $0.1 \%$ TFA and acetonitrile) of RPLC for each fraction were applied before analysis by MALDITOF/TOF. Peptides eluted from columns were directly mixed with $\alpha$-Cyano-4-hydroxycinnamic acid and spotted on a MALDI target plate with 768 spots followed by analysis by MS and MS/MS using ABI4800 MALDI-TOF/TOF.

\section{Data Analyses}

Peptide identifications-MS/MS spectra from LTQ were searched with SEQUEST [21] against a mouse protein database (the International Protein Index mouse protein database, version 3.13). The precursor mass tolerance is set as $3.0 \mathrm{Da}$. Other parameters of database searching are modified as follows: oxidized methionines (add Met with $16 \mathrm{Da}$ ), a (PNGase F-catalyzed) conversion of Asn to Asp (add Asn with $1 \mathrm{Da}$ ), and Cys modification (add cysteine with $57 \mathrm{Da}$ ). The output files were evaluated by INTERACT and PeptideProphet $[22,23]$. The criterion of PeptideProphet analysis is the probability score $\geq 0.9$ so that low probability protein identifications can be filtered out.

Identifying tissue-derived peptides in plasma from MALDI-TOF/TOF (ABI 4800) was performed using GPS Explorer software (version 3.6). MS/MS spectra were searched against NCBInr database. GPS searches were carried out at a 0.2-Da precursor mass tolerance, a 0.6-Da fragment mass tolerance, trypsin as digestion enzyme. In addition to the modifications for Met, Asp, and Cys that were used in LTQ MS/MS spectra analyses as described above, $\mathrm{N}$ termini of peptides and Lys are modified by succinic anhydride $\left(100 \mathrm{Da}\right.$ for $\mathrm{d} 0{ }^{13} \mathrm{C} 0,104 \mathrm{Da}$ for $\mathrm{d} 4{ }^{13} \mathrm{C} 0$, and $108 \mathrm{Da}$ for $\left.\mathrm{d} 4{ }^{13} \mathrm{C} 4\right)$.

\section{Results and Discussion}

Strategy of the Method

The objective of this study was to use N-linked glycopeptide isolation, isotopic labeling, and LC-MS to identify skin-cancer-related extracellular proteins and determine if these proteins could be detected in plasma from tumorbearing mice. This strategy is based on the fact that most extracellular proteins are glycoproteins, and extracellular proteins from cancer are most likely to be detected in plasma due to the fact that they are likely to be secreted by cells or shed from the cell surface to enter the blood stream.

The strategy is schematically illustrated in Fig. 1 and consists of four steps: (1) peptide extraction from tissue or plasma; (2) glycopeptide extraction: peptides that contain $\mathrm{N}$-linked carbohydrates in extracellular proteins were isolated in their de-glycosylated form using a recently described solid-phase capture-and-release method [9, 10]; (3) identification and quantification analysis of glycopeptides isolated from normal skin, papillomas, and carcinomas: isolated peptides were analyzed by LC-MS/MS, and the peptides were identified and quantified using a database search [21] and spectral count; (4) detection of tissuederived proteins in plasma. Glycopeptides from plasma samples taken from mice before and after development of skin tumors and tumor tissues were labeled with $\mathrm{d} 0{ }^{13} \mathrm{C} 0$, $\mathrm{d} 4{ }^{13} \mathrm{C} 0$, and $\mathrm{d} 4{ }^{13} \mathrm{C} 4$ succinic anhydride, respectively. The peptides containing $\mathrm{d} 4{ }^{13} \mathrm{C} 0$ and $\mathrm{d} 4{ }^{13} \mathrm{C} 4$ pairs indicated the tumor-derived peptides detected in plasma from tumorbearing mice, and they were selected for MS/MS analysis for peptide identifications.
Fig. 1 Flow chart for detection of tumor-specific proteins in plasma

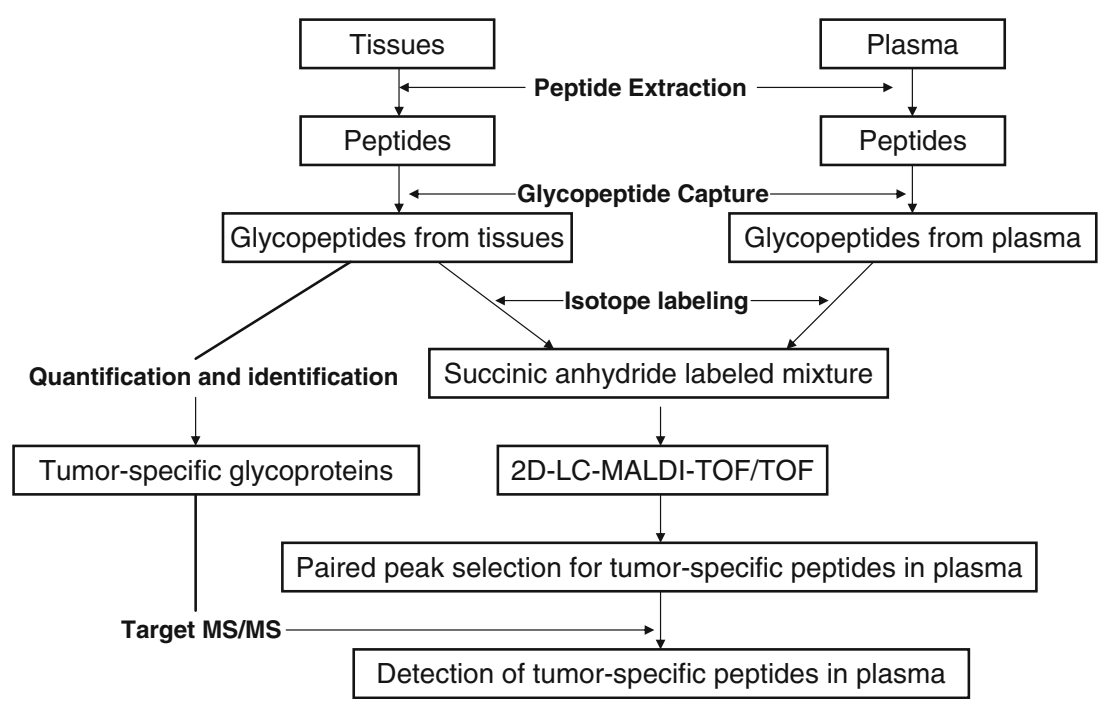


Identification of Proteins from a Mouse Model of Skin Cancer

To detect tumor-specific proteins in plasma, we first identified tumor-associated proteins from carcinomas and papillomas. These tumor-associated proteins are likely to be secreted or shed to the blood stream and fall into the detection range of current proteomic methodology.

To identify extracellular proteins from mouse skin tumors, four tissue samples each from normal skin, benign papillomas, and malignant carcinomas were collected to generate pooled normal, benign, and cancer tissues. Proteins were extracted from homogenized frozen tissues and digested to peptides. Glycopeptides were then captured using SPEG from each tissue. The N-linked glycopeptides were analyzed by LC-MS/MS by three repeated analyses for each sample. The MS/MS spectra were used to search protein databases using SEQUEST [21]. There were a total of 4,764 peptide identifications with PeptideProphet of at least 0.9 (with error rate of 0.007 ) from all the tissues. Ninety percent of these identifications (4,284 identifications) contained a consensus N-linked glycosylation motif (N-X-S/ $\mathrm{T}, \mathrm{X}$ is any amino acid except proline). These identifications were from 463 unique glycosylation sites representing 318 unique glycoproteins (Table 1). This indicated that the procedure was specific to N-linked glycoproteins. Therefore, we limited our subsequent analysis solely to the identified peptide sequences that contained at least one such consensus motif in order to reduce false positive rates. Since tissues are vascularized and some proteins identified from tissues are from contamination by common circulating blood proteins $[13,24]$, we next examined the glycoproteins identified from tissues to determine glycoproteins identified from tissues that were also identified from the normal mouse plasma $[10,25]$, and 59 glycoproteins were previously identified from normal mouse plasma and were not included for further study of skin cancer tissues.

To identify skin tumor-specific proteins, we compared the glycoproteins identified from normal skin, benign, and malignant tumors. Despite the same amount of glycopeptides from each tissue analyzed with the same procedures, the number of unique glycosites identified from different tissues was different. A total of 405 glycosites were identified in cancer tissue, while 252 in benign tissue and 112 in normal skin, when using PeptideProphet score of $\geq 0.9$. The number of glycoproteins identified from papillomas and carcinoma was higher than that of normal tissue. This could be caused by the increased expression of glycoproteins in tumor tissues. A similar observation was also reported from the proteomic analysis of tryptic peptides in a mouse breast cancer model [24].

To determine the glycoprotein changes associated with cancer development, we calculated the relative protein abundance using the number of redundant MS/MS spectra from the same glycoprotein in different tissues [26]. To eliminate the spectral count due to random events, only proteins identified with at least three spectra were included for quantitation. A number of proteins identified in this study were only detected in tumor tissues (benign or malignant) but not in normal tissues (the ratio of such proteins was arbitrary assigned to 100 , Table 2). Among the 111 proteins identified with spectral count ratio of at least three folds in cancer or benign tumor tissues comparing to normal tissues, 47 proteins (Table 2) were increased at least three folds in cancer tissues compared to benign tissues. Some of these have been reported to play roles in skin cancer development. These include known extracellular proteins such as thrombospondin, cathepsins, epidermal growth factor receptor, cell adhesion molecules, cadherins, integrins, tuberin, fibulin, and TGF $\beta$ receptor. Tenascin-C is an extracellular matrix glycoprotein and plays multiple functions in cell adhesion, migration, growth, and angiogenesis [27, 28]. Tenascin-C has many cell surface receptors, such as integrin and epidermal growth factor receptor, which may affect genome stability associated with interference with genome safeguard functions and escape from cell cycle checkpoints [28]. Tenascin-C has 20 potential N-linked glycosylation sites, but only one glycosylation site (LLQTAEHN\#ISGAER, Table 1) has been identified previously (Swiss-Prot Protein knowledgebase, http://us.expasy. org/sprot). In this study, eight N-linked glycosites including the previously identified site were identified in carcinomas (Table 1). They showed increased expression in carcinomas compared to papillomas (Table 2). This observation indicated that Tenascin- $\mathrm{C}$ might have increased its glycosylation or abundance during tumor development. In addition, 20 glycoproteins were identified in skin cancer only (Table 2), and these proteins might be used as protein markers to discriminate between the malignant and benign tumors. An example is Arylsulfatase B. In this study, Arylsulfatase B was identified three times only in malignant tissues with two unique glycosylation sites. Arylsulfatase B is a lysosomal enzyme and can degrade proteoglycans in the extracellular matrix and basement membrane. In this way, proteoglycans can obstruct the spread of cancer cells. Therefore, Arylsulfatase B could play a key role in accelerating cancer cell migration [29].

Here, we determined the relative abundance of glycosylated proteins using identified glycosylated peptides. However, glycosylation for individual glycosites from the same protein might be different and can be determined by quantitative analysis of each glycosite. In addition, changes in glycan structure that may be important to the disease cannot be determined by this method, and specific enrichment of glycopeptides with certain glycan structure is needed. 
Table 1 Identified N-linked glycoproteins and glycosites

\begin{tabular}{|c|c|c|c|}
\hline IPI & Protein name & $P$ & Identified sequences \\
\hline IPI00120245 & Integrin alpha- $\mathrm{V}$ & 1 & K.AN\#TTQPGIVEGGQVLK.C \\
\hline IPI00120245 & Integrin alpha-V & 1 & R.TAADATGLQPILNQFTPAN\#VSR.Q \\
\hline IPI00127447 & Lysosome membrane protein II & 1 & R.N\#QSVGDPNVDLIR.T \\
\hline IPI00127447 & Lysosome membrane protein II & 1 & T.GEDNYLN\#FSK.I \\
\hline IPI00127447 & Lysosome membrane protein II & 1 & R.TMVFPVMYLN\#ESVLIDK.E \\
\hline IPI00127447 & Lysosome membrane protein II & 1 & R.YKVPAEILAN\#TSENAGF.C \\
\hline IPI00322447 & RA175 & 1 & K.VSLTN\#VSISDEGR.Y \\
\hline IPI00322447 & RA175 & 1 & R.FQLLN\#FSSSELK.V \\
\hline IPI00118413 & Thrombospondin 1 & 1 & L.DNNVVN\#GSSPAIR.T \\
\hline IPI00118413 & Thrombospondin 1 & 1 & K.VSCPIMPCSN\#ATVPDGECCPR.C \\
\hline IPI00118413 & Thrombospondin 1 & 1 & W.PNENLVCVAN\#ATYHCK.K \\
\hline IPI00123678 & Cadherin-22 & 0.98 & R.ETAGWHN\#ITVLAMEADN.H \\
\hline IPI00154057 & Protocadherin 1 & 0.99 & N.DNAPFITAPSN\#TSHR.L \\
\hline IPI00126090 & Integrin alpha-3 & 1 & I.AMN\#YSLPLR.M \\
\hline IPI00126090 & Integrin alpha-3 & 1 & W.LECPLPDTSN\#ITN\#VTVK.A \\
\hline IPI00132474 & Integrin beta- 1 & 1 & R.NPCTSEQN\#CTSPFSYK.N \\
\hline IPI00132474 & Integrin beta- 1 & 1 & R.KEN\#SSEICSNNGECVCGQCVCR.K \\
\hline IPI00132474 & Integrin beta-1 & 1 & K.DTCAQECSHFN\#LTK.V \\
\hline IPI00227969 & Integrin alpha-6 & 1 & K.YQTLN\#CSVNVR.C \\
\hline IPI00227969 & Integrin alpha-6 & 0.91 & R.VEQKN\#NTFFDMNIF.E \\
\hline IPI00320605 & Integrin beta- 2 & 1 & K.LN\#FTGPGEPDSLR.C \\
\hline IPI00320605 & Integrin beta- 2 & 0.99 & Y.LRPGQAAAFN\#VTFR.R \\
\hline IPI00415773 & Integrin alpha-M & 1 & R.TPVLN\#CSVAVCK.R \\
\hline IPI00415773 & Integrin alpha-M & 1 & V.GGPQDFN\#MSVTLR.N \\
\hline IPI00415773 & Integrin alpha-M & 1 & R.LN\#YTLVGEPLR.S \\
\hline IPI00132067 & Fibulin-2 & 1 & Y.QLPGCHGN\#FSDAEEGDSER.Q \\
\hline IPI00132067 & Fibulin-2 & 1 & K.DLDECALGTHN\#CSEAETCHNIQGSFR.C \\
\hline IPI00132067 & Fibulin-2 & 1 & K.SCVAGVMGAKEGETCGAEDN\#DTCGVSLYK.A \\
\hline IPI00223769 & CD44 antigen & 1 & R.TEAADLCQAFN\#STLPTMDQMK.L \\
\hline IPI00110810 & Prostate stem cell antigen & 1 & R.DCLNVQN\#CSLDQHSCFTSR.I \\
\hline IPI00110852 & $\begin{array}{l}\text { Translocon-associated protein alpha, muscle } \\
\text { specific isoform }\end{array}$ & 1 & K.DLNGNVFQDAVFN\#QTVT.V \\
\hline IPI00110852 & $\begin{array}{l}\text { Translocon-associated protein alpha, muscle } \\
\text { specific isoform }\end{array}$ & 1 & R.YPQDYQFYIQN\#FTALPLNTVVPPQR.Q \\
\hline IPI00112326 & Epithelial membrane protein 1 & 1 & K.N\#CTGGNCDGSLSYGNEDAIK.A \\
\hline IPI00113480 & Myeloperoxidase & 1 & R.ALMPFDSLHDDPCLLTN\#R.S \\
\hline IPI00111013 & Cathepsin D & 1 & K.YYHGELSYLN\#VTR.K \\
\hline IPI00111013 & Cathepsin D & 1 & K.N\#GTSFDIHYGSGSL.S \\
\hline IPI00128154 & Cathepsin L & 1 & R.AEFAVAN\#DTGFVDIPQQEK.A \\
\hline IPI00403938 & Tenascin-C & 1 & L.EADTTQTVQN\#LTVPGGLR.S \\
\hline IPI00403938 & Tenascin-C & 1 & R.EPEIGNLN\#VSDVTPK.S \\
\hline IPI00403938 & Tenascin-C & 1 & R.LLQTAEHN\#ISGAER.T \\
\hline IPI00403938 & Tenascin-C & 1 & N.NVEAAQN\#LTVPGSLR.A \\
\hline IPI00403938 & Tenascin-C & 0.99 & N.NVETAHN\#FTVPGNLR.A \\
\hline IPI00403938 & Tenascin-C & 1 & R.ESGLN\#MTLPEENQPVVFNHIYNIK.L \\
\hline IPI00403938 & Tenascin-C & 1 & K.ASTEEVPSLEN\#LTVT.E \\
\hline IPI00403938 & Tenascin-C & 1 & R.LN\#YSLPTGQSMEVQLPK.D \\
\hline IPI00108535 & $\begin{array}{l}\text { Carcinoembryonic antigen-related cell adhesion } \\
\text { molecule } 1\end{array}$ & 1 & R.FVPNSNMN\#FTGQAYSGR.E \\
\hline IPI00108535 & $\begin{array}{l}\text { Carcinoembryonic antigen-related cell adhesion } \\
\text { molecule } 1\end{array}$ & 1 & K.N\#ITVLEPVTQPFLQVTN\#TTVK.E \\
\hline IPI00313428 & CEA-related cell adhesion molecule 2 & 1 & R.TLTLLN\#VTR.N \\
\hline IPI00122977 & Plasma protease $\mathrm{C} 1$ inhibitor & 1 & R.DTYVN\#ASQSLYGSSPR.V \\
\hline IPI00122977 & Plasma protease $\mathrm{C} 1$ inhibitor & 1 & K.VGQLQLSHN\#LSFVIVVPVFPK.H \\
\hline IPI00128689 & Collagen alpha $1(\mathrm{~V})$ chain & 1 & K.VYCN\#FTAGGSTCVFPDKK.S \\
\hline IPI00130249 & GPI-anchored metastasis-associated protein homolog & 1 & A.N\#VTVSLPVR.G \\
\hline
\end{tabular}


Table 1 (continued)

\begin{tabular}{|c|c|c|c|}
\hline IPI & Protein name & $P$ & Identified sequences \\
\hline IPI00130249 & GPI-anchored metastasis-associated protein homolog & 1 & K.CQGSMPPVVNCYN\#ASGR.V \\
\hline IPI00130486 & FK506-binding protein 9 & 1 & R.YHYN\#GTLLDGTLFDSSYSR.N \\
\hline IPI00130486 & FK506-binding protein 9 & 1 & R.YHYN\#GTFLDGTLFDSSHNR.M \\
\hline IPI00132600 & Niemann-Pick $\mathrm{C} 1$ protein & 1 & R.LYN\#VTHQFCN\#ASVMDPTCVR.C \\
\hline IPI00132600 & Niemann-Pick $\mathrm{C} 1$ protein & 1 & R.LIASN\#ITETMR.S \\
\hline IPI00131881 & ADAM 10 & 1 & R.IN\#TTSDEKDPTNPFR.F \\
\hline IPI00130342 & Lymphocyte antigen 6 complex locus G6C protein & 1 & K.LGLNYN\#TTCCDK.D \\
\hline IPI00130342 & Lymphocyte antigen 6 complex locus G6C protein & 1 & R.EVFN\#ETNHK.L \\
\hline IPI00133082 & CD177 antigen & 1 & K.VQGCMAQPDCNLLN\#GTQAI.G \\
\hline IPI00134549 & Lysosome-associated membrane glycoprotein 2 & 1 & A.LIVN\#LTDSK.G \\
\hline IPI00134549 & Lysosome-associated membrane glycoprotein 2 & 1 & K.VPFIFNINPATTN\#FTGSCQPQSAQLR.L \\
\hline IPI00134549 & Lysosome-associated membrane glycoprotein 2 & 1 & K.EVNVYMYLAN\#GSAFN\#ISNK.N \\
\hline IPI00121430 & Collagen alpha $1(\mathrm{XII})$ chain & 1 & K.EAGN\#ITTDGYEILGK.L \\
\hline IPI00122272 & Extracellular matrix protein 1 & 1 & K.QIPGLIQN\#MTVR.C \\
\hline IPI00122272 & Extracellular matrix protein 1 & 1 & R.NVALVAGDTGN\#ATGLGEQGPTR.G \\
\hline IPI00122493 & FK506-binding protein 10 & 1 & R.YHYN\#CSLLDGTR.L \\
\hline IPI00122493 & FK506-binding protein 10 & 1 & R.YHYN\#GSLMDGTLFDSSYSR.N \\
\hline IPI00123342 & Hypoxia up-regulated 1 & 1 & R.VFGSQN\#LTTVK.L \\
\hline IPI00123342 & Hypoxia up-regulated 1 & 1 & R.LSALDNLLN\#HSSIFLK.G \\
\hline IPI00123342 & Hypoxia up-regulated 1 & 1 & K.EN\#GTDAVQEEEESPAEGSK.D \\
\hline IPI00123831 & SDR1 protein & 1 & K.ENGVFEEISN\#SSGR.F \\
\hline IPI00123831 & SDR1 protein & 1 & R.FFITNKEN\#YTEL.S \\
\hline IPI00123831 & SDR1 protein & 1 & R.ESLLPVTLQCN\#LTSSSH.T \\
\hline IPI00224728 & Cd63 antigen & 1 & K.DRVPDSCCIN\#ITVGCGNDFK.E \\
\hline IPI00462199 & Basigin & 1 & K.TSDTGEEEAITN\#STEANGK.Y \\
\hline IPI00462199 & Basigin & 1 & K.TQLTCSLN\#SSGVDIVGHR.W \\
\hline IPI00462199 & Basigin & 1 & K.SQLTISNLDVNVDPGTYVCN\#ATNAQGTTR.E \\
\hline IPI00308609 & $\begin{array}{l}\text { VESICULAR INTEGRAL-MEMBRANE PROTEIN } \\
\text { VIP36 }\end{array}$ & 1 & R.VFPYISVMVNN\#GSLSYDHSK.D \\
\hline IPI00308990 & Monocyte differentiation antigen CD14 & 1 & R.N\#PSPDELPQVGN\#LSLK.G \\
\hline IPI00308785 & Prostaglandin $\mathrm{G} / \mathrm{H}$ synthase 2 & 1 & R.TGFYGEN\#CTTPEFLTR.I \\
\hline IPI00308971 & Cation-independent mannose-6-phosphate receptor & 1 & K.ISTN\#ITLVCKPGDLESAPVLR.A \\
\hline IPI00308971 & Cation-independent mannose-6-phosphate receptor & 1 & R.SLLEFN\#TTMGCQPSDSQHR.I \\
\hline IPI00124836 & Beta-sarcoglycan & 1 & R.ITSN\#ATSDLNIK.V \\
\hline IPI00124836 & Beta-sarcoglycan & 0.99 & I.ILN\#GTVMVSPTR.L \\
\hline IPI00122737 & $222 \mathrm{kDa}$ protein & 1 & R.QAEEAEEQANTN\#LSK.F \\
\hline IPI00122737 & $222 \mathrm{kDa}$ protein & 0.98 & R.VQLLHSQN\#TSLINQKK.K \\
\hline IPI00119063 & AM2 receptor & 1 & K.LTSCATN\#ASMCGDEAR.C \\
\hline IPI00119063 & AM2 receptor & 1 & K.LNLDGSN\#YTLLK.Q \\
\hline IPI00119063 & AM2 receptor & 1 & A.VAN\#DTNSCELSPCR.I \\
\hline IPI00119063 & AM2 receptor & 1 & R.MGCQHHCVPTPSGPTCYCN\#SSFQLE.A \\
\hline IPI00119063 & AM2 receptor & 0.99 & R.GVTHLN\#ISGLK.M \\
\hline IPI00119063 & AM2 receptor & 1 & R.FN\#STEYQVVTR.V \\
\hline IPI00124265 & Latent transforming growth factor beta binding protein 4 & 1 & R.N\#ATSVDSGAPGGAAPGGPGFR.A \\
\hline IPI00124265 & Latent transforming growth factor beta binding protein 4 & 1 & R.CTPACDPGYQPTPGGGCQDVDECRN\#R.S \\
\hline IPI00129304 & Collectin sub-family member 12 & 1 & R.HTDDLTSLN\#NTLVNIR.L \\
\hline IPI00129304 & Collectin sub-family member 13 & 1 & K.ETLQN\#NSFLITTVN\#K.T \\
\hline IPI00153959 & Stabilin-1 & 1 & H.ADLISN\#MSQDELAR.I \\
\hline IPI00153959 & Stabilin-1 & 1 & K.GFVDN\#MTLSGPDLELH.A \\
\hline IPI00316575 & Cathepsin K & 1 & Y.VGQDESCMYN\#ATAK.A \\
\hline IPI00126769 & Cathepsin F & 0.94 & K.VYIN\#DSVELSR.N \\
\hline IPI00121190 & Epidermal growth factor receptor & 1 & R.DIVQNVFMSN\#MSMDLQSHPSSCPK.C \\
\hline IPI00320420 & Clusterin & 1 & R.QELN\#DSLQVAER.L \\
\hline IPI00320420 & Clusterin & 0.99 & K.MLN\#TSSLLEQLNDQFNWVSQLAN\#LTQGEDK.Y \\
\hline IPI00406459 & Arylsulfatase B & 1 & H.EACAPIESLN\#GTR.C \\
\hline IPI00406459 & Arylsulfatase B & 1 & R.IYAGMVSLMDEAVGN\#VTK.A \\
\hline
\end{tabular}


Table 1 (continued)

\begin{tabular}{|c|c|c|c|}
\hline IPI & Protein name & $P$ & Identified sequences \\
\hline IPI00409393 & $\begin{array}{l}\text { Latent transforming growth factor beta binding protein, } \\
\text { isoform } 1 \mathrm{~L}\end{array}$ & 1 & R.YGQEQGTAPFQVSN\#HTGR.I \\
\hline IPI00409393 & $\begin{array}{l}\text { Latent transforming growth factor beta binding protein, } \\
\text { isoform } 1 \mathrm{~L}\end{array}$ & 1 & Y.NLNDASLCDNVLAPN\#VTK.Q \\
\hline IPI00409393 & $\begin{array}{l}\text { Latent transforming growth factor beta binding protein, } \\
\text { isoform } 1 \mathrm{~L}\end{array}$ & 0.91 & K.VCTN\#GSCTNLEGSYM.C \\
\hline IPI00108535 & $\begin{array}{l}\text { Carcinoembryonic antigen-related cell adhesion } \\
\text { molecule } 1\end{array}$ & 1 & R.EIIYSN\#GSLLFQMITMK.D \\
\hline IPI00117424 & Intercellular adhesion molecule 2 & 1 & K.IN\#CSTNCAAPDMGGLETPTNK.I \\
\hline IPI00122971 & $\begin{array}{l}\text { N-CAM } 180 \text { of Neural cell adhesion molecule } 1,180 \\
\mathrm{kDa} \text { isoform }\end{array}$ & 1 & R.DGQLLPSSN\#YSNIK.I \\
\hline IPI00406901 & Platelet/endothelial cell adhesion molecule & 1 & K.EETVLSQYQN\#FSK.I \\
\hline IPI00115976 & Integrin alpha-5 & 1 & K.VTGLSN\#CTSN\#YTPN.S \\
\hline IPI00313479 & Integrin beta 4 Isoform 2 & 1 & K.TCN\#CSTGSLSDTQPCLR.E \\
\hline IPI00466371 & Integrin alpha 1 & 1 & K.DSCESNQN\#ITCR.V \\
\hline IPI00230432 & Fibulin-1 & 0.98 & H.SYN\#SSLETIFIK.R \\
\hline IPI00119756 & OX-2 membrane glycoprotein & 1 & K.GTGTGIEN\#STESHFHSN\#GTTSVTSILR.V \\
\hline IPI00222589 & PTK7 protein tyrosine kinase 7 & 0.98 & R.MHIFQN\#GSLVIH.D \\
\hline IPI00314779 & TGF-beta receptor type III & 1 & R.AGVVVFN\#CSLR.Q \\
\hline IPI00112787 & Cell surface glycoprotein OX2 receptor & 1 & W.SPDGDCVTTSESHSN\#GTVTVR.S \\
\hline IPI00113528 & Transmembrane 9 superfamily protein member 3 & 1 & R.IVDVN\#LTSEGK.V \\
\hline IPI00114304 & Thrombospondin-3 & 1 & R.LGFLGN\#QSQGCVPAR.T \\
\hline IPI00119809 & Mama protein & 1 & R.ALGYEN\#ATQALGR.A \\
\hline IPI00119809 & Mama protein & 1 & K.GLN\#LTEDTYKPR.L \\
\hline IPI00469218 & Lysosomal membrane glycoprotein 1 & 1 & R.LN\#MTLPDALVPTFSISN\#HSLK.A \\
\hline IPI00469218 & Lysosomal membrane glycoprotein 2 & 1 & K.N\#VTVVLR.D \\
\hline IPI00120025 & Similar to KALLIKREIN 9 & 1 & R.LTPAVQPLN\#LTESRPPVGTQ.C \\
\hline IPI00338790 & Glandular kallikrein KLK13 & 1 & K.ILN\#GTN\#GTSGFLPGGYTCLPH.S \\
\hline IPI00116993 & Tuberin & 0.94 & A.PKQGLN\#NSPPVK.E \\
\hline IPI00111550 & Mucin and cadherin-like protein & 1 & R.VTN\#SSEFMMNK.D \\
\hline IPI00108041 & Stromal interaction molecule 1 & 1 & R.LAVTN\#TTMTGTVLK.M \\
\hline IPI00108328 & $\begin{array}{l}\text { Methylated-DNA- protein-cysteine methyltransferase } \\
\text { containing protein }\end{array}$ & 0.93 & M.ETTSLLLCIGN\#NSSGIRSRHR.S \\
\hline IPI00108811 & Glucosylceramidase & 1 & R.DLGPALAN\#SSHDVK.L \\
\hline IPI00109281 & Enabled protein homolog & 1 & W.ERTNTMN\#GSK.S \\
\hline IPI00109612 & Laminin, beta 2 & 1 & L.ASGN\#VSGGVCDGCQHNTAGR.H \\
\hline IPI00109727 & Thy-1 membrane glycoprotein & 1 & K.VLTLAN\#FTTK.D \\
\hline IPI00109908 & Ig gamma-2A chain $\mathrm{C}$ region, membrane-bound form & 1 & R.EDYN\#STLR.V \\
\hline IPI00111014 & Elongation of very long chain fatty acids protein 4 & 1 & T.AFN\#DTVEFYR.W \\
\hline IPI00111115 & $\begin{array}{l}\text { Similar to METASTASIS-ASSOCIATED GPI- } \\
\text { ANCHORED PROTEIN }\end{array}$ & 1 & R.MNIGN\#FSVPVYIR.T \\
\hline IPI00111960 & Lysosomal alpha-glucosidase & 1 & R.GVFITN\#ETGQPLIGK.V \\
\hline IPI00112176 & Copper homeostasis protein cutC homolog & 0.94 & R.N\#SSVAMGASLAHSEYSLK.V \\
\hline IPI00113057 & Plasma kallikrein & 1 & K.LQTPLN\#YTEFQKPICLPSK.A \\
\hline IPI00113797 & Napsin A & 0.96 & W.FN\#LTGQDYVIK.I \\
\hline IPI00113824 & $\begin{array}{l}\text { Basement membrane-specific heparan sulfate } \\
\text { proteoglycan core protein }\end{array}$ & 0.98 & K.LTVPSSQN\#SSFR.L \\
\hline IPI00113824 & $\begin{array}{l}\text { Basement membrane-specific heparan sulfate } \\
\text { proteoglycan core protein }\end{array}$ & 1 & R.SLTQGSLIVGNLAPVN\#GTSQGK.F \\
\hline IPI00113824 & $\begin{array}{l}\text { Basement membrane-specific heparan sulfate } \\
\text { proteoglycan core protein }\end{array}$ & 1 & R.VAQQDSGQYICN\#ATNSAGH.T \\
\hline IPI00113853 & Desmocollin-3 & 0.99 & K.AN\#FTILK.G \\
\hline IPI00113854 & Eosinophil peroxidase & 0.99 & F.DNLHEDPCLLTN\#R.S \\
\hline IPI00114065 & Complement factor B & 1 & K.IVLDPSGSMNIYLVLDGSDSIGSSN\#FTGAK.R \\
\hline IPI00114065 & Complement factor B & 0.94 & R.SPFYN\#LSDQI.S \\
\hline IPI00114206 & Prothrombin & 1 & R.WVLTAAHCILYPPWDKN\#FTENDLLVR.I \\
\hline
\end{tabular}


Table 1 (continued)

\begin{tabular}{|c|c|c|c|}
\hline IPI & Protein name & $P$ & Identified sequences \\
\hline IPI00114206 & Prothrombin & 1 & R.ITDNMFCAGFKVN\#DTK.R \\
\hline IPI00400016 & Laminin gamma-1 chain & 1 & K.LLNN\#LTSIK.I \\
\hline IPI00400016 & Laminin gamma-1 chain & 1 & R.TLAGEN\#QTALEIEELNR.K \\
\hline IPI00400016 & Laminin gamma-1 chain & 1 & L.SYGQN\#LSFSFR.V \\
\hline IPI00400016 & Laminin gamma-1 chain & 1 & R.KYEQAKN\#ISQDLEKQ.A \\
\hline IPI00317340 & Lactotransferrin & 1 & I.PMGLLAN\#QTR.S \\
\hline IPI00317340 & Lactotransferrin & 1 & K.N\#SSNFHLNQLQGLR.S \\
\hline IPI00113539 & Fibronectin & 1 & R.DQCIVDDITYNVN\#DTFHK.R \\
\hline IPI00113539 & Fibronectin & 1 & K.LDAPTNLQFVN\#ETDR.T \\
\hline IPI00113539 & Fibronectin & 1 & R.HEEGHMLN\#CTCFGQGR.G \\
\hline IPI00119818 & Inter alpha-trypsin inhibitor, heavy chain 4 & 1 & K.AFITN\#FSMIIDGVTYPGVVK.E \\
\hline IPI00119818 & Inter alpha-trypsin inhibitor, heavy chain 5 & 1 & R.GLMLLLN\#DTQHFSNNVK.G \\
\hline IPI00114256 & Synaptophysin-like protein & 1 & K.N\#QTVTATFGYPFR.L \\
\hline IPI00114319 & Extracellular superoxide dismutase $[\mathrm{Cu}-\mathrm{Zn}]$ & 1 & R.LEAYFSLEGFPAEQN\#ASNR.A \\
\hline IPI00114641 & CD98 heavy chain & 1 & K.LMNAPLYLAEWQN\#ITK.N \\
\hline IPI00114810 & Suppressor of tumorigenicity 14 & 0.99 & R.VIN\#QTTCEDLMPQQITPR.M \\
\hline IPI00114958 & HMW of Kininogen-1 & 1 & K.HSIEHFNN\#NTDHSHLFTLR.K \\
\hline IPI00114958 & HMW of Kininogen-1 & 1 & T.YTIVQTN\#CSK.E \\
\hline IPI00114958 & HMW of Kininogen-1 & 1 & K.IAN\#FSQSCTLYSGDDLVEALPKPCPGCPR.D \\
\hline IPI00115089 & Ectonucleoside triphosphate diphosphohydrolase 2 & 1 & R.LLN\#LTSPEATAK.V \\
\hline IPI00115516 & EMILIN-1 & 1 & R.FN\#STLGPSEEQEK.N \\
\hline IPI00115530 & Beta-hexosaminidase beta chain & 1 & K.TQVFGPVDPTVN\#TTYA.F \\
\hline IPI00115762 & Neural cell adhesion molecule L1 & 1 & K.EQLFFN\#LSDPELR.T \\
\hline IPI00115817 & PREDICTED: similar to ribosomal protein L21 & 0.95 & K.TGRVYN\#VTQHAMGIIVNK.Q \\
\hline IPI00115854 & TROP2 protein & 1 & R.AFN\#HSDLDSELR.R \\
\hline IPI00116105 & Corticosteroid-binding globulin & 1 & K.DLFTN\#QSDFADTTK.D \\
\hline IPI00116105 & Corticosteroid-binding globulin & 1 & R.EEDFYVN\#ETSTVK.V \\
\hline IPI00116105 & Corticosteroid-binding globulin & 1 & K.VPMMVQSGN\#ISYFR.D \\
\hline IPI00116105 & Corticosteroid-binding globulin & 1 & R.GSTQYLENLGFN\#MSK.M \\
\hline IPI00116599 & p130Cas-associated protein & 0.92 & R.RQVDEGMWPPPNNLLN\#QSPK.K \\
\hline IPI00116913 & Laminin alpha-5 chain & 1 & R.QLLAN\#SSALEETILGHQGR.L \\
\hline IPI00116913 & Laminin alpha-5 chain & 1 & H.N\#FSGCISNVFVQR.L \\
\hline IPI00116945 & Complement factor D & 1 & K.LSQN\#ASLGPHVRPLPLQYEDK.E \\
\hline IPI00117093 & Laminin beta-3 chain & 1 & R.QTACTPGDCPGELCPQDN\#GTACGSHCR.G \\
\hline IPI00117140 & Fc receptor, IgG, low affinity IIb & 1 & R.YHHYSSN\#FSIPK.A \\
\hline IPI00117735 & Myelin P0 protein & 1 & K.DGSIVIHNLDYSDN\#GTFTCDVK.N \\
\hline IPI00117831 & Ceruloplasmin & 1 & K.EYEGAVYPDN\#TTDFQR.A \\
\hline IPI00117857 & Alpha-1-antitrypsin 1-6 & 1 & K.GDTHTQILEGLQFN\#LTQTSEADIHK.S \\
\hline IPI00117932 & Paired amphipathic helix protein $\operatorname{Sin} 3 a$ & 0.96 & P.DAN\#SSVLLSKTTAEK.V \\
\hline IPI00117957 & Asporin & 1 & R.ITDIEN\#GTFANIPR.V \\
\hline IPI00118011 & mannosidase, beta $\mathrm{A}$, lysosomal & 0.97 & V.AEILFNN\#VTIGK.T \\
\hline IPI00118130 & Alpha-1-acid glycoprotein 1 & 1 & R.ESQTIGDQCVYN\#STHLGFQR.E \\
\hline IPI00118130 & Alpha-1-acid glycoprotein 1 & 1 & R.QAIQTMQSEFFYLTTNLIN\#DTIELR.E \\
\hline IPI00118130 & Alpha-1-acid glycoprotein 1 & 1 & R.EN\#GTFSKYEGGVETFAHLIVLR.K \\
\hline IPI00118191 & Receptor-type tyrosine-protein phosphatase N2 & 0.98 & K.VSANIQN\#MTTADVIK.A \\
\hline IPI00118385 & Glutamate [NMDA] receptor subunit zeta 1 & 1 & K.VICTGPN\#DTSPGSPR.H \\
\hline IPI00118437 & Complement component $\mathrm{C} 8$ gamma chain homolog & 1 & R.EAN\#LTEDQILFFPK.Y \\
\hline IPI00119004 & $\begin{array}{l}\text { Hypothetical Lipolytic enzyme, G-D-S-L containing } \\
\text { protein }\end{array}$ & 0.91 & R.KGPGMENPVAVTIFFGAN\#DSSLK.D \\
\hline IPI00119299 & Leukemia inhibitory factor receptor & 1 & K.VVLAGSN\#MTICCMSPTK.V \\
\hline IPI00119299 & Leukemia inhibitory factor receptor & 1 & R.IEGLTN\#ETYR.L \\
\hline IPI00119299 & Leukemia inhibitory factor receptor & 1 & R.LGVQMHPGQEIHN\#FTLTGR.N \\
\hline IPI00119522 & Carboxypeptidase $\mathrm{N}$, polypeptide 2 homolog & 1 & R.LQDLEITGSPVSN\#LSAHIFSN\#LSSLEK.L \\
\hline IPI00119627 & Insulin receptor substrate 1 & 0.93 & K.LLPCTGDYMN\#MSPVGDSN\#TS.S \\
\hline IPI00120187 & Fibromodulin & 0.97 & R.VPNNALEGLEN\#LT.A \\
\hline IPI00120751 & Proton myo-inositol transporter homolog & 1 & K.IN\#GSAVIDSSCVPVNK.A \\
\hline
\end{tabular}


Table 1 (continued)

\begin{tabular}{|c|c|c|c|}
\hline IPI & Protein name & $P$ & Identified sequences \\
\hline IPI00120769 & $\begin{array}{l}\text { Solute carrier family } 29 \text { (nucleoside transporters), } \\
\text { member } 1\end{array}$ & 1 & R.LDVSQN\#VSSDTDQSCESTK.A \\
\hline IPI00120848 & Mimecan & 0.95 & I.SSLTDDTFCKAN\#DTR.Y \\
\hline IPI00121038 & Versican core protein & 1 & R.FEN\#QTCFPLPDSR.F \\
\hline IPI00121120 & Procollagen, type V, alpha 2 & 1 & K.EASQN\#LTYICR.N \\
\hline IPI00121312 & MFIRE1 & 1 & K.IDLTDFEKN\#SSFA.Q \\
\hline IPI00121362 & F11r protein & 1 & R.AFMN\#SSFTIDPK.S \\
\hline IPI00121418 & Retinoblastoma-associated protein & 0.97 & K.QLEN\#DTRIIEVLCKEHECNIDEVKN.V \\
\hline IPI00121550 & Sodium/potassium-transporting ATPase beta- 1 chain & 1 & K.LDWLGN\#CSGLNDDSYGYR.E \\
\hline IPI00121634 & High-affinity cationic amino acid transporter- 1 & 0.94 & K.FLAKINN\#RTKTPVIATVTSGAIAAVM.A \\
\hline IPI00122293 & Prolargin & 1 & R.VPVIPPRIHYLYLQNNFITELPLESFQN\#ATGLR.W \\
\hline IPI00122302 & Neutrophil elastase homolog & 0.91 & R.LGTNRPSPSVLQELN\#VT.V \\
\hline IPI00122368 & $\mathrm{P} 2 \mathrm{X} 4 \mathrm{c}$ receptor subunit & 1 & K.TSICDSDAN\#CTLGSSDTHSSGIGTGR.C \\
\hline IPI00122438 & Fibrillin-1 & 1 & K.AWGTPCELCPSVN\#TSEYK.I \\
\hline IPI00122438 & Fibrillin-1 & 1 & V.DTDECSVGNPCGN\#GTCK.N \\
\hline IPI00122438 & Fibrillin-1 & 1 & V.N\#VTDYCQLVR.Y \\
\hline IPI00122438 & Fibrillin-1 & 1 & R.NYYADN\#QTCDGELLFN\#MTK.K \\
\hline IPI00122438 & Fibrillin-1 & 1 & R.N\#CTDIDECR.I \\
\hline IPI00123194 & Biglycan & 1 & R.MIEN\#GSLSFLPTLR.E \\
\hline IPI00123196 & Decorin & 1 & K.LGLSFNSITVMEN\#GSLANVPHLR.E \\
\hline IPI00123196 & Decorin & 1 & K.YIQVVYLHNNN\#ISAVGQNDFCR.A \\
\hline IPI00123223 & Murinoglobulin-1 & 1 & R.NYEVQLFHVN\#ATVTEEGTGLEFSR.S \\
\hline IPI00123223 & Murinoglobulin-1 & 1 & R.N\#ASFVYTK.A \\
\hline IPI00123824 & Amiloride-sensitive sodium channel beta-subunit & 1 & K.GEPYSPCTMN\#GSDVAIK.N \\
\hline IPI00123957 & Cd97 protein & 1 & R.DFNPATVN\#YTIQK.L \\
\hline IPI00123996 & Neuropilin-1 & 1 & K.RGPECSQN\#YTAPTGVIK.S \\
\hline IPI00124283 & Macrophage scavenger receptor types I and II & 1 & R.VLNN\#ITNDLR.L \\
\hline IPI00124640 & $\begin{array}{l}\text { Osteoclast-like cell cDNA, clone:I420031M06 } \\
\text { product:granulin }\end{array}$ & 1 & K.SDTPCDDFTRCPTN\#NTCCK.L \\
\hline IPI00124830 & Leukocyte surface antigen CD47 & 1 & I.EFTSCN\#ETVVIPCIVR.N \\
\hline IPI00125058 & Laminin alpha-3 chain & 1 & K.IESINQQLLPLGN\#ISDNVDR.I \\
\hline IPI00125058 & Laminin alpha-3 chain & 0.99 & K.TTFNLN\#TTEVEPCRR.R \\
\hline IPI00125266 & Acid ceramidase & 1 & R.SVLEN\#TTSYEEAK.N \\
\hline IPI00125293 & Eosinophil cationic protein 1 & 0.97 & R.VHITVCN\#ITSR.A \\
\hline IPI00125310 & Complement $\mathrm{C} 1 \mathrm{q}$ subcomponent, A chain & 1 & K.VLTNQESPYQN\#HTGR.F \\
\hline IPI00125325 & Peroxisomal 2,4-dienoyl-CoA reductase & 0.96 & F.RDHGGVIVN\#ITATLSMR.G \\
\hline IPI00125514 & Ectonucleoside triphosphate diphosphohydrolase 5 & 1 & R.GYLTSFEMFN\#STFK.L \\
\hline IPI00125877 & Hypothetical protein & 1 & N.YQN\#NTEVIQGIR.T \\
\hline IPI00125877 & Hypothetical protein & 1 & R.GLTFLKN\#VSSTCAASPSTDILTFTIPPSFADIFLSK.S \\
\hline IPI00126050 & Plasma glutamate carboxypeptidase & 1 & K.EVMNLLQPLN\#VTK.V \\
\hline IPI00126186 & Macrophage mannose receptor 1 & 1 & R.TSYCN\#ESFYFLCK.K \\
\hline IPI00126194 & Alpha-2-macroglobulin & 1 & K.N\#ITSVVSPLGYLSIFTTDEHGLAN\#ISIDTSN\#FTAPFLR.V \\
\hline IPI00126194 & Alpha-2-macroglobulin & 1 & R.IN\#VSYTGERPSSNMVIVDVK.M \\
\hline IPI00126194 & Alpha-2-macroglobulin & 1 & Y.LN\#ETQQLTEAIK.S \\
\hline IPI00126194 & Alpha-2-macroglobulin & 1 & K.VN\#LSFPSAQSLPASDTHLK.V \\
\hline IPI00126316 & Mast cell carboxypeptidase A & 1 & R.NQN\#STCIGTDLNR.N \\
\hline IPI00126834 & Vascular cell adhesion protein 1 & 1 & K.ETTIWVSPSPILEEGSPVN\#LTCSSDGIPAPK.I \\
\hline IPI00127280 & Myeloid bactenecin & 1 & K.DCDFLEDGEERN\#CTGK.F \\
\hline IPI00127352 & AMBP protein & 1 & K.EDSCQLN\#YSEGPCLGMQER.Y \\
\hline IPI00127560 & Transthyretin & 1 & K.TLGISPFHEFADVVFTAN\#DSGHR.H \\
\hline IPI00127672 & PREDICTED: hypothetical protein LOC66967 & 1 & K.LLPAFN\#TTSGLPYPR.I \\
\hline IPI00127856 & Alpha-1-acid glycoprotein 2 & 1 & R.EYHTIDDHCVYN\#STHLGIQR.E \\
\hline IPI00127856 & Alpha-1-acid glycoprotein 2 & 1 & D.PITN\#ETLSWLSDK.W \\
\hline IPI00127933 & Androgen binding protein alpha & 1 & R.KVDLFLN\#GTTEEY.V \\
\hline IPI00128249 & Alpha-2-HS-glycoprotein & 1 & R.RPFGVVYEMEVDTLETTCHALDPTPLAN\#CSVR.Q \\
\hline IPI00128249 & Alpha-2-HS-glycoprotein & 1 & R.CPLLTPFN\#DTNVVHTVNTALAAFNTQNN\#GTYFK.L \\
\hline
\end{tabular}


Table 1 (continued)

\begin{tabular}{|c|c|c|c|}
\hline IPI & Protein name & $P$ & Identified sequences \\
\hline IPI00128484 & Hemopexin & 1 & R.VAEVEN\#GTKPD.S \\
\hline IPI00128484 & Hemopexin & 1 & R.SWSTVGN\#CTAALR.W \\
\hline IPI00128484 & Hemopexin & 1 & K.SLGPNTCSSN\#GSSLYFIHGPNLYCYSSIDK.L \\
\hline IPI00128484 & Hemopexin & 1 & M.DHN\#GTMLFFK.G \\
\hline IPI00128905 & Golgi phosphoprotein 2 & 1 & K.AVLVNN\#ITTGEK.L \\
\hline IPI00128989 & Vacuolar ATP synthase subunit S1 & 1 & A.IHPPVSYN\#DTAPR.I \\
\hline IPI00129158 & $\begin{array}{l}\text { Tyrosine-protein phosphatase non-receptor type } \\
\text { substrate } 1\end{array}$ & 1 & R.GIAN\#LSNFIR.V \\
\hline IPI00129243 & Gamma-glutamyl hydrolase & 1 & K.LPLN\#FTEGAR.K \\
\hline IPI00129243 & Gamma-glutamyl hydrolase & 0.99 & L.ALEN\#LTANFHK.W \\
\hline IPI00129250 & Leucine-rich alpha-2-glycoprotein & 1 & L.SVEFSN\#LTQLPAAALQGCPGLR.E \\
\hline IPI00129250 & Leucine-rich alpha-2-glycoprotein & 1 & K.MFSQN\#DTR.C \\
\hline IPI00129359 & zinc finger protein 68 & 0.97 & K.ELAGIGNTCN\#VSTNH.I \\
\hline IPI00129965 & PREDICTED: similar to alpha-1-B glycoprotein & 1 & K.LLFVGPQHAGN\#YSCR.Y \\
\hline IPI00129966 & PREDICTED: similar to alpha-1-B glycoprotein & 0.99 & R.VYQPGN\#YSCSYQTHGECTSSTPSR.I \\
\hline IPI00129968 & Embigin & 1 & K.DDEPLETTGDFN\#TTK.M \\
\hline IPI00130010 & Complement factor $\mathrm{H}$ & 1 & K.DNSCVDPPHVPN\#ATIVTR.T \\
\hline IPI00130010 & Complement factor $\mathrm{H}$ & 1 & K.LTEFTHN\#STMDYK.C \\
\hline IPI00130010 & Complement factor $\mathrm{H}$ & 1 & R.TKCIN\#GTINYPTCV.- \\
\hline IPI00130015 & Dipeptidyl-peptidase I & 1 & R.ILTN\#NSQTPILSPQEVVSCSPYAQGCDGGFPYLIAGK.Y \\
\hline IPI00130483 & $\mathrm{KH}$ domain RNA binding protein QKI-5A & 0.96 & R.KDMYN\#DTLN\#GSTEK.R \\
\hline IPI00130627 & Legumain & 0.97 & Y.DDIANSEEN\#PTPGVVINRPN\#GTDVYK.G \\
\hline IPI00130630 & Glutamate carboxypeptidase II & 1 & K.VPYNVGPGFAGN\#FSTQK.V \\
\hline IPI00130654 & Afamin & 1 & P.TKPQDVDHFN\#ATQK.F \\
\hline IPI00130654 & Afamin & 1 & L.ADLVLGELCGVNTN\#R.T \\
\hline IPI00130661 & Tripeptidyl-peptidase I & 0.97 & K.DVGSGTTN\#NSQACAQFLEQYFHNSDLTEFMR.L \\
\hline IPI00130661 & Tripeptidyl-peptidase I & 1 & K.SSSHLPPSSYFN\#ASGR.A \\
\hline IPI00131114 & Type VI collagen alpha 3 subunit & 1 & R.GPPGVN\#GTQGFQGCPGQR.G \\
\hline IPI00131114 & Type VI collagen alpha 3 subunit & 1 & R.ALN\#GSALYTGSSLDFVR.N \\
\hline IPI00131114 & Type VI collagen alpha 3 subunit & 1 & R.QLINALQIN\#NTAVGHALVLPAR.R \\
\hline IPI00131137 & $9 \mathrm{kDa}$ protein & 0.96 & K.GKAN\#ASEDANNPAENGDAK.T \\
\hline IPI00131209 & Keratin intermediate filament $16 \mathrm{a}$ & 1 & R.KTEELNKEVASNSDLIQSN\#R.S \\
\hline IPI00131366 & Keratin, type II cytoskeletal 6B & 1 & R.VPGLN\#RSGFSSVSVCR.S \\
\hline IPI00131526 & CD209 antigen-like protein B & 1 & R.IPIFQGQN\#ESIQEK.I \\
\hline IPI00131830 & Serine protease inhibitor $\mathrm{A} 3 \mathrm{~K}$ & 1 & K.NLINDYVSN\#QTQGMIK.E \\
\hline IPI00131830 & Serine protease inhibitor $\mathrm{A} 3 \mathrm{~K}$ & 1 & K.YTGN\#ASALLILPDQGR.M \\
\hline IPI00131951 & Serpin A12 & 0.91 & L.SLGAQN\#STLEEIR.E \\
\hline IPI00133035 & $\mathrm{NAD}(\mathrm{P})(+)$-arginine ADP-ribosyltransferase & 1 & R.LGN\#FTLAYSAKPETADNQR.V \\
\hline IPI00133035 & $\mathrm{NAD}(\mathrm{P})(+)$-arginine ADP-ribosyltransferase & 1 & K.GTSNDLVLQSIN\#STCSYYECAFLGGLK.T \\
\hline IPI00133172 & Serpin B11 & 1 & K.N\#SSECSQVGVMHPDFR.A \\
\hline IPI00133257 & Hematopoietic progenitor cell antigen CD34 & 1 & M.VLAN\#STELPSK.L \\
\hline IPI00133751 & Microfibril-associated glycoprotein 4 & 1 & R.VDLEDFEN\#NTAYAK.Y \\
\hline IPI00133751 & Microfibril-associated glycoprotein 4 & 1 & R.FN\#GSVSFFR.G \\
\hline IPI00134191 & $\begin{array}{l}\text { Solute carrier family } 2 \text {, facilitated glucose transporter } \\
\text { member } 3\end{array}$ & 1 & K.DFLN\#YTLEER.L \\
\hline IPI00134483 & Lectin lambda & 1 & R.PGACTN\#ITMGVVCK.L \\
\hline IPI00134483 & Lectin lambda & 1 & R.VTPVCN\#ASLPAQR.W \\
\hline IPI00134547 & Zinc finger autosomal protein & 0.98 & V.ELLDPN\#NSICVPREK.M \\
\hline IPI00134652 & Type VII collagen & 1 & K.LQILN\#ASSDVLR.V \\
\hline IPI00134808 & C4b-binding protein & 1 & R.LACLN\#GTVLR.G \\
\hline IPI00134808 & C4b-binding protein & 1 & R.LVGSPFIGCTVVN\#K.T \\
\hline IPI00136642 & Antithrombin-III & 1 & K.LGACN\#DTLK.Q \\
\hline IPI00136902 & Piccolo protein & 0.97 & Y.RRQISAVQPSIIN\#LSAASSLGTPVTMDSK.T \\
\hline IPI00136925 & Immunoglobulin $\mathrm{J}$ chain & 1 & R.EN\#ISDPTSPLR.R \\
\hline IPI00137177 & Lysosomal protective protein & 1 & R.LDPPCTN\#TTAPSNYLNNPYVR.K \\
\hline IPI00137987 & Zinc-alpha-2-glycoprotein & 1 & K.DTTGSHTFQGMFGCEITNN\#R.S \\
\hline
\end{tabular}


Table 1 (continued)

\begin{tabular}{|c|c|c|c|}
\hline IPI & Protein name & $P$ & Identified sequences \\
\hline IPI00138342 & Liver carboxylesterase $\mathrm{N}$ & 1 & R.FHSELN\#ISESMIPAVIEK.Y \\
\hline IPI00139788 & Serotransferrin & 1 & K.N\#STLCDLCIGPLK.C \\
\hline IPI00153187 & Sulfatase modifying factor 1 & 1 & K.FVN\#STGYLTEAEK.F \\
\hline IPI00153202 & Angiotensin-converting enzyme 2 & 0.99 & Y.FFVTSPQN\#VSDVIPR.S \\
\hline IPI00153258 & Protein Z-dependent protease inhibitor & 1 & R.ASQQLSN\#ETSSFGFNLLR.K \\
\hline IPI00153548 & Hypothetical protein & 0.9 & C.QFGVGTFANVFLFVYN\#FSPISTGSK.Q \\
\hline IPI00169815 & Procollagen, type VI, alpha 2 & 1 & R.GTFTDCALAN\#MTQQIR.Q \\
\hline IPI00169815 & Procollagen, type VI, alpha 2 & 1 & I.GYTN\#FTLEK.N \\
\hline IPI00169815 & Procollagen, type VI, alpha 2 & 1 & R.MALLQYGSQNQQQVAFPLTYN\#VTTIHEALER.A \\
\hline IPI00169815 & Procollagen, type VI, alpha 2 & 1 & R.N\#MTLFSDLVAEK.F \\
\hline IPI00169858 & Hypothetical protein LOC435366 & 0.97 & R.HERN\#QSAEKPSEYTQHGKAFALHAHSHAQ.R \\
\hline IPI00169896 & Choline transporter-like protein 2 & 1 & K.TCNPETFPLRN\#ESLQCPTAR.C \\
\hline IPI00221418 & Hypothetical Phospholipase D/Transphosphatidylase & 1 & K.VFIVPVGN\#HSNIPFSR.V \\
\hline IPI00221426 & Glucosamine (N-acetyl)-6-sulfatase & 1 & K.YYN\#YTLSINGK.A \\
\hline IPI00221456 & Synaptic vesicle glycoprotein $2 \mathrm{~b}$ & 0.96 & K.KVLSMSLAIN\#ASFASLSSFVQGY.G \\
\hline IPI00221833 & Hypothetical Zinc finger, $\mathrm{C} 2 \mathrm{H} 2$ type containing protein & 0.96 & D.WMPNN\#HSVILIDDFESPQK.L \\
\hline IPI00223446 & Laminin alpha-4 chain & 1 & R.HVTDMN\#STIHLLR.T \\
\hline IPI00223987 & $\begin{array}{l}\text { Insulin-regulated membrane aminopeptidase IRAP } \\
\text { homolog }\end{array}$ & 1 & R.MAFDLIDYLKN\#ETHTAPI.T \\
\hline IPI00224456 & Sarcalumenin & 1 & A.PLIN\#VTEPPR.V \\
\hline IPI00224456 & Sarcalumenin & 1 & K.TN\#VSKFDLPNR.E \\
\hline IPI00224584 & Calsequestrin 2 & 1 & K.IDLFKPQIGVVN\#VTDADSI.W \\
\hline IPI00224654 & Hypothetical protein & 1 & R.AYIQDFQEFSKN\#ISIMLGR.C \\
\hline IPI00225355 & Target of Nesh-SH3 variant 1 & 0.99 & K.VHIN\#TTSDSILLK.F \\
\hline IPI00226310 & $\begin{array}{l}\text { Hypothetical von Willebrand factor type A domain } \\
\text { containing protein }\end{array}$ & 1 & R.DLSVFAPN\#MTEIIK.D \\
\hline IPI00226310 & $\begin{array}{l}\text { Hypothetical von Willebrand factor type A domain } \\
\text { containing protein }\end{array}$ & 1 & K.LGN\#FSELATHN\#QTFLK.K \\
\hline IPI00226310 & $\begin{array}{l}\text { Hypothetical von Willebrand factor type A domain } \\
\text { containing protein }\end{array}$ & 0.99 & L.LDMAIN\#GSQEDLDHLK.A \\
\hline IPI00226790 & GPI transamidase component PIG-T & 0.92 & L.GLAN\#DTDDYFLR.Y \\
\hline IPI00226932 & $\begin{array}{l}\text { Quinoprotein alcohol dehydrogenase structure } \\
\text { containing protein }\end{array}$ & 1 & R.FINYN\#QTVSR.M \\
\hline IPI00227834 & Inter-alpha trypsin inhibitor, heavy chain 2 & 1 & K.GAFISN\#FTMTVNGMTFTSSIK.E \\
\hline IPI00227857 & Hepatocyte growth factor activator & 1 & R.FCNIVPTEHCFLGN\#GTEYR.G \\
\hline IPI00229117 & Tenascin-N & 1 & Y.ILTYQFPN\#GTVK.E \\
\hline IPI00230289 & Excitatory amino acid transporter 2 & 1 & K.VLVAPPSEEAN\#TTK.A \\
\hline IPI00266902 & PREDICTED: similar to type V P-type ATPase isoform 3 & 0.99 & K.VCDPNSDVCN\#TTR.S \\
\hline IPI00271166 & Huntington disease gene homolog & 0.94 & R.GYSLLPSITDVTMENN\#LSR.V \\
\hline IPI00271262 & Murinoglobulin-2 & 1 & K.ELIFYYLVMAQGSIIQTGN\#HTHQVEPGEAPVK.G \\
\hline IPI00272381 & Proline 4-hydroxylase, alpha 1 & 1 & K.DMSDGFISN\#LTIQR.Q \\
\hline IPI00279010 & Lu protein & 1 & F.VFLN\#SSSTVVN\#CSAR.G \\
\hline IPI00279051 & RIKEN cDNA A930025J12 & 1 & R.LFQN\#CSELYK.A \\
\hline IPI00279079 & Fibrinogen beta chain & 1 & K.GTAGNALMDGASQLVGEN\#R.T \\
\hline IPI00281188 & $140 \mathrm{kDa}$ protein & 0.99 & K.VLEPPHIN\#GSEGPGEV.S \\
\hline IPI00281344 & $\begin{array}{l}\text { Hypothetical Glycosyl transferase, family } 8 \text { containing } \\
\text { protein }\end{array}$ & 0.93 & R.TGVNSGVMLMN\#MTR.M \\
\hline IPI00308213 & Ig gamma-1 chain $\mathrm{C}$ region, membrane-bound form & 1 & R.EEQFN\#STFR.S \\
\hline IPI00308658 & Olfactomedin-like protein 3 & 1 & K.IYVLDGTQN\#DTAFVFPR.L \\
\hline IPI00309068 & E130014G12 product:Kaiso protein & 1 & K.EDLPSN\#NT.A \\
\hline IPI00309214 & Serum amyloid P-component & 1 & K.LIPHLEKPLQN\#FTLCFR.T \\
\hline IPI00309230 & Beta-glucuronidase & 0.98 & R.ITIAIN\#NTLTPH.T \\
\hline IPI00309999 & Laminin alpha-2 chain & 1 & R.LEQMTMNIN\#LTGPLPAPYK.I \\
\hline IPI00309999 & Laminin alpha-2 chain & 1 & K.LN\#ETLGNQDK.T \\
\hline IPI00309999 & Laminin alpha-2 chain & 1 & R.ICNQN\#SSNPYQR.H \\
\hline IPI00309999 & Laminin alpha-2 chain & 1 & K.VFQAESHAAQLN\#DSSAVLDGILDEAK.N \\
\hline
\end{tabular}


Table 1 (continued)

\begin{tabular}{|c|c|c|c|}
\hline IPI & Protein name & $P$ & Identified sequences \\
\hline IPI00309999 & Laminin alpha-2 chain & 1 & K.VCN\#CSTVGSLASQCNVNTGQCSCHPK.F \\
\hline IPI00309999 & Laminin alpha-2 chain & 1 & K.ILYGLEN\#TTQELK.H \\
\hline IPI00309999 & Laminin alpha-2 chain & 1 & K.YIGGGVCIN\#CTHNTA.G \\
\hline IPI00309999 & Laminin alpha-2 chain & 1 & Y.VGGLPIN\#YTTR.R \\
\hline IPI00309999 & Laminin alpha-2 chain & 1 & L.NLASNALITTN\#ATCGEK.G \\
\hline IPI00310049 & Carboxypeptidase B2 & 1 & K.EVHFFVN\#ASDVDSVK.A \\
\hline IPI00311808 & Transmembrane glycoprotein NMB & 1 & R.DLPIVFDVLIHDPSHFLN\#DSAISYK.W \\
\hline IPI00313900 & Lumican & 1 & K.LHINYNN\#LTESVGPLPK.S \\
\hline IPI00313900 & Lumican & 1 & R.LSHNELADSGVPGNSFN\#ISSLLELDLSYNK.L \\
\hline IPI00313900 & Lumican & 1 & K.AFEN\#VTDLQWLILDHNLLENSK.I \\
\hline IPI00313900 & Lumican & 1 & K.LGSFDGLVN\#LTFIYLQHNQLK.E \\
\hline IPI00316329 & keratin complex 2 , basic, gene 1 & 1 & R.MSGECTPN\#VSVSVSTSHTSMSGSSSR.G \\
\hline IPI00318012 & T-cell immunomodulatory protein & 1 & V.PCNN\#ASCEEVHR.M \\
\hline IPI00318595 & Adipocyte-derived leucine aminopeptidase & 1 & L.N\#SSHPVSTPVENPAQIR.E \\
\hline IPI00319814 & Suprabasal-specific protein suprabasin & 1 & K.EANQLLN\#GSHQGQGGYGGQHGGAATT.T \\
\hline IPI00320204 & RIKEN cDNA 2210023G05 & 1 & R.DGTSQPAICPQN\#VTMNMEGLK.E \\
\hline IPI00320675 & Complement factor I & 1 & R.WGEVDLIGN\#CSQFYPDR.Y \\
\hline IPI00320675 & Complement factor I & 1 & N.FN\#VSLIYGR.T \\
\hline IPI00321190 & Sulfated glycoprotein 1 & 1 & K.TN\#SSFIQGFVDHVKEDCDR.L \\
\hline IPI00321190 & Sulfated glycoprotein 1 & 1 & K.DN\#ATQEEILHYLEK.T \\
\hline IPI00322304 & Histidine-rich glycoprotein HRG & 1 & R.LPPLNIGEVLTLPEANFPSFSLPNCN\#R.S \\
\hline IPI00322463 & Beta-2-glycoprotein I & 1 & K.DYRPSAGN\#NSLYQDTVVFK.C \\
\hline IPI00322463 & Beta-2-glycoprotein I & 1 & K.N\#ISFACNPGFFLN\#GTSSSK.C \\
\hline IPI00322575 & ATP-binding cassette transporter sub-family A member $8 \mathrm{a}$ & 0.95 & K.NTQNILVQN\#LSGGQKRK.L \\
\hline IPI00330747 & 5730439E10Rik protein & 0.94 & R.YLMGN\#NSSEDSFLTANTVQPLAETGLQLSK.R \\
\hline IPI00331214 & Platelet glycoprotein IV & 1 & R.QFWIFDVQNPDDVAKN\#SSK.I \\
\hline IPI00331214 & Platelet glycoprotein IV & 1 & K.DPFLSLVPYPISTTVGVFYPYN\#DTVDGVYK.V \\
\hline IPI00331214 & Platelet glycoprotein IV & 1 & K.VISNN\#CTSYGVLDIGK.C \\
\hline IPI00331214 & Platelet glycoprotein IV & 1 & K.RPYIVPILWLN\#ETGTIGDEK.A \\
\hline IPI00331214 & Platelet glycoprotein IV & 1 & K.EN\#ITQDPEDH.T \\
\hline IPI00331214 & Platelet glycoprotein IV & 1 & R.N\#LSYWPSYCDMIN\#GTDAASFPPFVEK.S \\
\hline IPI00331259 & Desmoglein-1 gamma & 1 & K.LN\#ATDADEPNNLNSMIAFK.I \\
\hline IPI00331617 & Hypothetical olfactomedin-like domain containing protein & 1 & R.VDKLEEEVSKN\#LTK.E \\
\hline IPI00338565 & Mutant fibrillin-1 & 1 & R.VLPFN\#VTDYCQLVR.Y \\
\hline IPI00338785 & $\begin{array}{l}\text { CDNA, clone:M5C1012G13 product:laminin B1 } \\
\text { subunit } 1\end{array}$ & 1 & K.MEMPSTPQQLQN\#LTEDIR.E \\
\hline IPI00338785 & $\begin{array}{l}\text { CDNA, clone:M5C1012G13 product:laminin B1 } \\
\text { subunit } 1\end{array}$ & 1 & K.QADEDIQGTQNLLTSIESETAASEETLTN\#ASQR.I \\
\hline IPI00338785 & $\begin{array}{l}\text { CDNA, clone:M5C1012G13 product:laminin B1 } \\
\text { subunit } 1\end{array}$ & 1 & L.ATGN\#VSGGVCDNCQHNTMGR.N \\
\hline IPI00338785 & $\begin{array}{l}\text { CDNA, clone:M5C1012G13 product:laminin B1 } \\
\text { subunit } 1\end{array}$ & 1 & R.VN\#ASTTDPN\#STVEQSALTR.D \\
\hline IPI00338785 & $\begin{array}{l}\text { CDNA, clone:M5C1012G13 product:laminin B1 } \\
\text { subunit } 1\end{array}$ & 1 & K.LTDTASQSN\#STAGELGALQAEAESLDK.T \\
\hline IPI00339885 & Collagen alpha $1(\mathrm{VI})$ chain & 1 & R.AALQFLQN\#YTVL.A \\
\hline IPI00339885 & Collagen alpha $1(\mathrm{VI})$ chain & 1 & L.DDGFLKN\#ITAQICIDKK.C \\
\hline IPI00340463 & PREDICTED: similar to hypothetical protein A030003A19 & 1 & K.LLNDYVSN\#QTQGMIK.E \\
\hline IPI00346978 & Spink5 protein & 0.99 & E.TNKNSASRSN\#GTGSATGKDVCDQFR.S \\
\hline IPI00346978 & Spink5 protein & 0.96 & K.GNQDPCMKFQAQMKN\#GTLTCPK.G \\
\hline IPI00348602 & Weakly similar to Zinnc finger protein GLI4 & 0.98 & R.FRN\#SSNLARHR.R \\
\hline IPI00350715 & PREDICTED: similar to protocadherin 9 & 1 & R.IDPVTGN\#ITLEEKPAPTDVGLHR.L \\
\hline IPI00355606 & PREDICTED: expressed sequence AL022779 & 0.96 & M.QN\#NSVFGDLK.S \\
\hline IPI00378430 & Ortholog of human Ras association & 0.94 & R.QETNMAN\#FSYR.F \\
\hline IPI00381122 & Weakly similar to Tiarin & 1 & K.IN\#LTTNVVDVNRPLPL.A \\
\hline IPI00403586 & Hypothetical Lipolytic enzymes & 1 & M.IVNN\#HTSLDVER.A \\
\hline IPI00405742 & Plexin B2 & 0.95 & K.QDLALSGN\#LSSLYAMTQDK.V \\
\hline
\end{tabular}


Table 1 (continued)

\begin{tabular}{|c|c|c|c|}
\hline IPI & Protein name & $P$ & Identified sequences \\
\hline IPI00406434 & Mini-agrin & 1 & K.NELMLN\#SSLMR.I \\
\hline IPI00407222 & PREDICTED: similar to human KIAA1815 protein & 0.99 & H.IPEIN\#DTIR.A \\
\hline IPI00408344 & PREDICTED: similar to solute carrier family 4 member 11 & 0.94 & R.EDSLGDEVFDTVN\#SSIVSGESIR.F \\
\hline IPI00409148 & Haptoglobin & 1 & K.NLFLN\#HSETASAK.D \\
\hline IPI00409148 & Haptoglobin & 1 & K.N\#LTSPVGVQPILNEHTFCAGLTK.Y \\
\hline IPI00409148 & Haptoglobin & 1 & K.CVVHYEN\#STVPEK.K \\
\hline IPI00409148 & Haptoglobin & 1 & K.VVLHPN\#HSVVDIGLIK.L \\
\hline IPI00410951 & Thyroxine-binding globulin homolog & 1 & K.VTTCHLPQQN\#ATLYK.M \\
\hline IPI00420489 & Von Willebrand factor & 1 & V.LEGSDEVGEANFN\#K.S \\
\hline IPI00420955 & Sortilin 1 & 1 & K.DITNLIN\#NTFIR.T \\
\hline IPI00453607 & Killer cell inhibitory receptor-like protein p91A & 1 & R.LSVLPSPVVTAGGN\#MTLH.C \\
\hline IPI00458917 & Sodium/glucose cotransporter 1 & 1 & K.VSNGN\#FTAK.E \\
\hline IPI00459432 & Kidney predominant protein & 1 & S.ADFQGRPVDDPTGAFAN\#GSLTFK.V \\
\hline IPI00460063 & Prenylcysteine oxidase & 1 & K.GELN\#STLFSSRPK.D \\
\hline IPI00461281 & NudC domain containing protein 2 & 1 & K.ENPGFDFSGAEISGN\#YTK.G \\
\hline IPI00462999 & Ahi-1 isoform III & 0.92 & D.EFVNTEN\#NSSR.K \\
\hline IPI00463311 & PREDICTED: similar to RIKEN cDNA E330026B02 & 0.99 & R.DLGMFAPN\#MTR.I \\
\hline IPI00467180 & Translocon-associated protein beta subunit & 1 & R.IAPASN\#VSHTVVLRPLK.A \\
\hline IPI00467944 & $61 \mathrm{kDa}$ protein & 1 & K.VVN\#VSELYGTPCTK.R \\
\hline IPI00468097 & $340 \mathrm{kDa}$ protein & 1 & R.NLQVYN\#ATSNSLTVK.W \\
\hline IPI00469000 & Zinc transporter SLC39A6 & 0.98 & R.NTNDNIQECFN\#TTK.L \\
\hline IPI00469387 & GUGU alpha & 1 & R.VLYLPAYN\#CTLRPVSK.R \\
\hline IPI00469387 & GUGU alpha & 1 & R.SPPAPPLPQRPLSPLHPLGCN\#DSEVLAVAGFALQNINR.D \\
\hline IPI00469542 & Histidine-rich calcium-binding protein & 1 & R.EVGEEN\#VSEEVFR.G \\
\hline IPI00469839 & $19 \mathrm{kDa}$ protein & 0.91 & K.TRTIDVVYN\#ASNNELVCTK.T \\
\hline IPI00471081 & RIKEN cDNA $1100001 \mathrm{H} 23$ & 1 & K.NGDAYGYYN\#DSIK.T \\
\hline IPI00471273 & Apoptosis-related protein 3 & 1 & A.LPEICTLCPGGMHN\#LSR.V \\
\hline IPI00473625 & PREDICTED: laminin, alpha 3 & 0.98 & R.FN\#ISTPAFQGCMK.N \\
\hline IPI00473830 & Biliary glycoprotein & 1 & R.MTLSQN\#NSILR.I \\
\hline IPI00475154 & $\begin{array}{l}\text { Dolichyl-diphosphooligosaccharide-protein } \\
\text { glycosyltransferase } 63 \mathrm{kDa}\end{array}$ & 1 & Q.VLSGCEISVSN\#ETK.E \\
\hline IPI00475157 & Serpina1b protein & 1 & R.ELVHQSN\#TSNIFFSPVSIATAFAMLSLGSK.G \\
\hline IPI00475157 & Serpina1b protein & 1 & N.ASAVFLLPEDGK.M \\
\hline IPI00551354 & PREDICTED: ring finger and $\mathrm{KH}$ domain containing 3 & 0.91 & R.N\#GSGGGGGGGGGGGGGGSGGGETLDDQR.A \\
\hline IPI00553278 & H-2D cell surface glycoprotein & 1 & R.NLLGYYN\#QSAGGSHTLQQM.S \\
\hline IPI00554833 & Eosinophil-associated ribonuclease 12 & 1 & V.GVCGN\#PSGLCSDN\#ISQNCHN\#SSSR.V \\
\hline IPI00606550 & Ig gamma-2B chain $\mathrm{C}$ region, membrane-bound form & 1 & R.EDYN\#STIR.V \\
\hline IPI00607976 & $\begin{array}{l}\text { Serine (or cysteine) proteinase inhibitor, clade } \mathrm{A}, \\
\text { member } 3 \mathrm{~A}\end{array}$ & 1 & K.FN\#LTETPEADIH.Q \\
\hline IPI00621319 & $43 \mathrm{kDa}$ protein & 0.92 & K.RLFLLDLLN\#ATGK.D \\
\hline IPI00624663 & Pzp protein & 0.99 & K.ACVSLNHVN\#ETVM.L \\
\hline IPI00624761 & $44 \mathrm{kDa}$ protein & 1 & R.PVDDPTGAFAN\#GSLTFK.V \\
\hline IPI00626315 & $38 \mathrm{kDa}$ protein & 0.94 & P.PSSTDLLWSILN\#ASALALLYKTQRDN\#ASESK.D \\
\hline IPI00627061 & MKIAA4087 protein & 0.94 & R.CNIN\#GSFSEICHTR.T \\
\hline IPI00649090 & $\begin{array}{l}\text { Adult male thymus cDNA, clone:5830446P09 product: } \\
\text { CD72 antigen }\end{array}$ & 0.96 & V.GSEQPTATWSSVN\#SSALRQIPR.C \\
\hline IPI00649281 & $52 \mathrm{kDa}$ protein & 0.98 & R.YHYN\#GTLLDGTAFDNSYSR.N \\
\hline IPI00654271 & Myosin light chain, regulatory $\mathrm{B}$ & 0.91 & K.N\#PTDAYLDAMMNEAPAPIN\#FTMFL.T \\
\hline IPI00654907 & Hypothetical protein CEACAM1/2sec & 1 & R.FHVHQPVTQPFLQVTN\#TTVK.E \\
\hline
\end{tabular}

P: peptide probability

N\#: N-linked glycosylation site 
Table 2 Glycoproteins upregulated in skin tumors

\begin{tabular}{|c|c|c|c|c|c|c|c|c|c|}
\hline IPI & Protein name & Protein location & $\mathrm{Ca}$ & $\mathrm{Pa}$ & $\mathrm{Nr}$ & Total & $\mathrm{Ca} / \mathrm{Nr}$ & $\mathrm{Pa} / \mathrm{Nr}$ & $\mathrm{Ca} / \mathrm{Pa}$ \\
\hline IPI00119063 & AM2 receptor & Transmembrane & 17 & 0 & 0 & 17 & 100.0 & 0.0 & 100.0 \\
\hline IPI00381122 & Weakly similar to Tiarin & Cell Surface & 11 & 0 & 0 & 11 & 100.0 & 0.0 & 100.0 \\
\hline IPI00308971 & Cation-independent mannose-6-phosphate receptor & Transmembrane & 8 & 0 & 0 & 8 & 100.0 & 0.0 & 100.0 \\
\hline IPI00124265 & $\begin{array}{l}\text { Latent transforming growth factor beta binding } \\
\text { protein } 4\end{array}$ & Secreted & 7 & 0 & 0 & 7 & 100.0 & 0.0 & 100.0 \\
\hline IPI00129304 & Collectin sub-family member 12 & Transmembrane & 7 & 0 & 0 & 7 & 100.0 & 0.0 & 100.0 \\
\hline IPI00129968 & Embigin & Transmembrane & 7 & 0 & 0 & 7 & 100.0 & 0.0 & 100.0 \\
\hline IPI00153959 & Stabilin-1 & Transmembrane & 5 & 0 & 0 & 5 & 100.0 & 0.0 & 100.0 \\
\hline IPI00316575 & Cathepsin K & Cell Surface & 4 & 0 & 0 & 4 & 100.0 & 0.0 & 100.0 \\
\hline IPI00321190 & Sulfated glycoprotein 1 & Secreted & 4 & 0 & 0 & 4 & 100.0 & 0.0 & 100.0 \\
\hline IPI00475154 & $\begin{array}{l}\text { Dolichyl-diphosphooligosaccharide-protein } \\
\text { glycosyltransferase } 63 \mathrm{kDa}\end{array}$ & Transmembrane & 4 & 0 & 0 & 4 & 100.0 & 0.0 & 100.0 \\
\hline IPI00308785 & Prostaglandin $\mathrm{G} / \mathrm{H}$ synthase 2 & Secreted & 3 & 0 & 0 & 3 & 100.0 & 0.0 & 100.0 \\
\hline IPI00122737 & $222 \mathrm{kDa}$ protein & Intracellular & 3 & 0 & 0 & 3 & 100.0 & 0.0 & 100.0 \\
\hline IPI00406459 & Arylsulfatase B & Secreted & 3 & 0 & 0 & 3 & 100.0 & 0.0 & 100.0 \\
\hline IPI00409393 & $\begin{array}{l}\text { Latent transforming growth factor beta binding } \\
\text { protein, isoform } 1 \mathrm{~L}\end{array}$ & Secreted & 3 & 0 & 0 & 3 & 100.0 & 0.0 & 100.0 \\
\hline IPI00119809 & Mama protein & Secreted & 3 & 0 & 0 & 3 & 100.0 & 0.0 & 100.0 \\
\hline IPI00111960 & Lysosomal alpha-glucosidase & Transmembrane & 3 & 0 & 0 & 3 & 100.0 & 0.0 & 100.0 \\
\hline IPI00118011 & Mannosidase, beta A, lysosomal & Secreted & 3 & 0 & 0 & 3 & 100.0 & 0.0 & 100.0 \\
\hline IPI00121120 & Procollagen, type V, alpha 2 & Secreted & 3 & 0 & 0 & 3 & 100.0 & 0.0 & 100.0 \\
\hline IPI00129158 & $\begin{array}{l}\text { Tyrosine-protein phosphatase non-receptor type } \\
\text { substrate } 1\end{array}$ & Transmembrane & 3 & 0 & 0 & 3 & 100.0 & 0.0 & 100.0 \\
\hline IPI00120769 & $\begin{array}{l}\text { Solute carrier family } 29 \text { (nucleoside transporters), } \\
\text { member } 1\end{array}$ & Transmembrane & 5 & 0 & 1 & 6 & 5.0 & 0.0 & 100.0 \\
\hline IPI00415773 & Integrin alpha-M & Transmembrane & 34 & 3 & 1 & 38 & 34.0 & 3.0 & 11.3 \\
\hline IPI00338785 & $\begin{array}{l}\text { cDNA, clone:M5C1012G13 product:laminin B1 } \\
\text { subunit } 1\end{array}$ & Intracellular & 9 & 1 & 3 & 13 & 3.0 & 0.3 & 9.0 \\
\hline IPI00130627 & Legumain & Secreted & 17 & 2 & 0 & 19 & 100.0 & 100.0 & 8.5 \\
\hline IPI00113480 & Myeloperoxidase & Secreted & 8 & 1 & 0 & 9 & 100.0 & 100.0 & 8.0 \\
\hline IPI00113824 & $\begin{array}{l}\text { Basement membrane-specific heparan sulfate } \\
\text { proteoglycan core protein }\end{array}$ & Cell Surface & 15 & 2 & 5 & 22 & 3.0 & 0.4 & 7.5 \\
\hline IPI00124830 & Leukocyte surface antigen CD47 & Transmembrane & 7 & 1 & 1 & 9 & 7.0 & 1.0 & 7.0 \\
\hline IPI00320605 & Integrin beta- 2 & Transmembrane & 20 & 3 & 0 & 23 & 100.0 & 100.0 & 6.7 \\
\hline IPI00308990 & Monocyte differentiation antigen CD14 & Cell Surface & 13 & 2 & 0 & 15 & 100.0 & 100.0 & 6.5 \\
\hline IPI00133082 & CD177 antigen & Secreted & 6 & 1 & 0 & 7 & 100.0 & 100.0 & 6.0 \\
\hline IPI00130486 & FK506-binding protein 9 & Cell Surface & 5 & 1 & 0 & 6 & 100.0 & 100.0 & 5.0 \\
\hline IPI00308609 & $\begin{array}{l}\text { VESICULAR INTEGRAL-MEMBRANE PROTEIN } \\
\text { VIP36 }\end{array}$ & Transmembrane & 5 & 1 & 0 & 6 & 100.0 & 100.0 & 5.0 \\
\hline IPI00403938 & Tenascin-C & Cell Surface & 133 & 29 & 0 & 162 & 100.0 & 100.0 & 4.6 \\
\hline IPI00462199 & Basigin & Transmembrane & 13 & 3 & 0 & 16 & 100.0 & 100.0 & 4.3 \\
\hline IPI00120245 & Integrin alpha-V & Transmembrane & 8 & 2 & 0 & 10 & 100.0 & 100.0 & 4.0 \\
\hline IPI00120245 & Integrin alpha-V & Transmembrane & 8 & 2 & 0 & 10 & 100.0 & 100.0 & 4.0 \\
\hline IPI00110852 & $\begin{array}{l}\text { Translocon-associated protein alpha, muscle specific } \\
\text { isoform }\end{array}$ & Cell Surface & 4 & 1 & 0 & 5 & 100.0 & 100.0 & 4.0 \\
\hline IPI00125266 & Acid ceramidase & Secreted & 4 & 1 & 0 & 5 & 100.0 & 100.0 & 4.0 \\
\hline IPI00121038 & Versican core protein & Cell Surface & 11 & 3 & 2 & 16 & 5.5 & 1.5 & 3.7 \\
\hline IPI00124283 & Macrophage scavenger receptor types I and II & Transmembrane & 7 & 2 & 0 & 9 & 100.0 & 100.0 & 3.5 \\
\hline IPI00132067 & Fibulin-2 & Secreted & 31 & 9 & 0 & 40 & 100.0 & 100.0 & 3.4 \\
\hline IPI00223769 & CD44 antigen & Transmembrane & 10 & 3 & 0 & 13 & 100.0 & 100.0 & 3.3 \\
\hline IPI00127447 & Lysosome membrane protein II & Transmembrane & 32 & 10 & 0 & 42 & 100.0 & 100.0 & 3.2 \\
\hline IPI00322447 & RA175 & Transmembrane & 6 & 2 & 0 & 8 & 100.0 & 100.0 & 3.0 \\
\hline IPI00154057 & Protocadherin 1 & Cell Surface & 3 & 1 & 0 & 4 & 100.0 & 100.0 & 3.0 \\
\hline IPI00121312 & MFIRE1 & Secreted & 3 & 1 & 0 & 4 & 100.0 & 100.0 & 3.0 \\
\hline IPI00124640 & Osteoclast-like cell cDNA, clone:I420031M06 product:granulin & Secreted & 3 & 1 & 0 & 4 & 100.0 & 100.0 & 3.0 \\
\hline IPI00134483 & Lectin lambda & Cell Surface & 3 & 1 & 1 & 5 & 3.0 & 1.0 & 3.0 \\
\hline
\end{tabular}


Table 2 (continued)

\begin{tabular}{|c|c|c|c|c|c|c|c|c|c|}
\hline IPI & Protein name & Protein location & $\mathrm{Ca}$ & $\mathrm{Pa}$ & $\mathrm{Nr}$ & Total & $\mathrm{Ca} / \mathrm{Nr}$ & $\mathrm{Pa} / \mathrm{Nr}$ & $\mathrm{Ca} / \mathrm{Pa}$ \\
\hline IPI00272381 & Proline 4-hydroxylase, alpha 1 & Secreted & 17 & 6 & 0 & 23 & 100.0 & 100.0 & 2.8 \\
\hline IPI00122493 & FK506-binding protein 10 & Secreted & 7 & 3 & 0 & 10 & 100.0 & 100.0 & 2.3 \\
\hline IPI00123831 & SDR1 protein & Transmembrane & 11 & 5 & 0 & 16 & 100.0 & 100.0 & 2.2 \\
\hline IPI00224728 & Cd63 antigen & Transmembrane & 8 & 4 & 0 & 12 & 100.0 & 100.0 & 2.0 \\
\hline IPI00128689 & Collagen alpha $1(\mathrm{~V})$ chain & Secreted & 6 & 3 & 0 & 9 & 100.0 & 100.0 & 2.0 \\
\hline IPI00125877 & Hypothetical protein & Transmembrane & 6 & 3 & 0 & 9 & 100.0 & 100.0 & 2.0 \\
\hline IPI00130015 & Dipeptidyl-peptidase I & Secreted & 4 & 2 & 0 & 6 & 100.0 & 100.0 & 2.0 \\
\hline IPI00318012 & T-cell immunomodulatory protein & Transmembrane & 4 & 2 & 0 & 6 & 100.0 & 100.0 & 2.0 \\
\hline IPI00123678 & Cadherin-22 & Transmembrane & 2 & 1 & 0 & 3 & 100.0 & 100.0 & 2.0 \\
\hline IPI00126316 & Mast cell carboxypeptidase A & Secreted & 2 & 1 & 0 & 3 & 100.0 & 100.0 & 2.0 \\
\hline IPI00130661 & Tripeptidyl-peptidase I & Cell Surface & 2 & 1 & 0 & 3 & 100.0 & 100.0 & 2.0 \\
\hline IPI00131366 & Keratin, type II cytoskeletal 6B & Transmembrane & 2 & 1 & 0 & 3 & 100.0 & 100.0 & 2.0 \\
\hline IPI00221418 & hypothetical Phospholipase D/Transphosphatidylase & Transmembrane & 2 & 1 & 0 & 3 & 100.0 & 100.0 & 2.0 \\
\hline IPI00279051 & RIKEN cDNA A930025J12 & Transmembrane & 2 & 1 & 0 & 3 & 100.0 & 100.0 & 2.0 \\
\hline IPI00554833 & Eosinophil-associated ribonuclease 12 & Secreted & 2 & 1 & 0 & 3 & 100.0 & 100.0 & 2.0 \\
\hline IPI00127280 & Myeloid bactenecin & Secreted & 43 & 22 & 0 & 65 & 100.0 & 100.0 & 2.0 \\
\hline IPI00118413 & Thrombospondin 1 & Secreted & 20 & 11 & 0 & 31 & 100.0 & 100.0 & 1.8 \\
\hline IPI00127352 & AMBP protein & Secreted & 22 & 14 & 0 & 36 & 100.0 & 100.0 & 1.6 \\
\hline IPI00132600 & Niemann-Pick $\mathrm{C} 1$ protein & Transmembrane & 3 & 2 & 0 & 5 & 100.0 & 100.0 & 1.5 \\
\hline IPI00137177 & Lysosomal protective protein & Secreted & 3 & 2 & 0 & 5 & 100.0 & 100.0 & 1.5 \\
\hline IPI00132474 & Integrin beta-1 & Transmembrane & 18 & 13 & 1 & 32 & 18.0 & 13.0 & 1.4 \\
\hline IPI00123342 & Hypoxia up-regulated 1 & Secreted & 19 & 14 & 0 & 33 & 100.0 & 100.0 & 1.4 \\
\hline IPI00126090 & Integrin alpha-3 & Transmembrane & 4 & 3 & 0 & 7 & 100.0 & 100.0 & 1.3 \\
\hline IPI00131881 & ADAM 10 & Cell Surface & 4 & 3 & 0 & 7 & 100.0 & 100.0 & 1.3 \\
\hline IPI00406434 & Mini-agrin & Secreted & 4 & 3 & 0 & 7 & 100.0 & 100.0 & 1.3 \\
\hline IPI00410951 & Thyroxine-binding globulin homolog & Secreted & 4 & 3 & 0 & 7 & 100.0 & 100.0 & 1.3 \\
\hline IPI00125058 & Laminin alpha-3 chain & Secreted & 9 & 7 & 1 & 17 & 9.0 & 7.0 & 1.3 \\
\hline IPI00112326 & Epithelial membrane protein 1 & Transmembrane & 6 & 5 & 0 & 11 & 100.0 & 100.0 & 1.2 \\
\hline IPI00128154 & Cathepsin L & Secreted & 23 & 20 & 0 & 43 & 100.0 & 100.0 & 1.2 \\
\hline IPI00121362 & F11r protein & Transmembrane & 9 & 9 & 0 & 18 & 100.0 & 100.0 & 1.0 \\
\hline IPI00108535 & $\begin{array}{l}\text { Carcinoembryonic antigen-related cell adhesion } \\
\text { molecule } 1\end{array}$ & Cell Surface & 7 & 7 & 0 & 14 & 100.0 & 100.0 & 1.0 \\
\hline IPI00407222 & PREDICTED: similar to human KIAA1815 protein & Transmembrane & 6 & 6 & 0 & 12 & 100.0 & 100.0 & 1.0 \\
\hline IPI00128989 & Vacuolar ATP synthase subunit S1 & Transmembrane & 5 & 5 & 0 & 10 & 100.0 & 100.0 & 1.0 \\
\hline IPI00471081 & RIKEN cDNA $1100001 \mathrm{H} 23$ & Cell Surface & 5 & 5 & 0 & 10 & 100.0 & 100.0 & 1.0 \\
\hline IPI00226932 & $\begin{array}{l}\text { Quinoprotein alcohol dehydrogenase structure } \\
\text { containing protein }\end{array}$ & Secreted & 4 & 4 & 0 & 8 & 100.0 & 100.0 & 1.0 \\
\hline IPI00127672 & PREDICTED: hypothetical protein LOC66967 & Secreted & 2 & 2 & 0 & 4 & 100.0 & 100.0 & 1.0 \\
\hline IPI00346978 & Spink5 protein & Secreted & 2 & 2 & 0 & 4 & 100.0 & 100.0 & 1.0 \\
\hline IPI00469387 & GUGU alpha & Secreted & 23 & 23 & 3 & 49 & 7.7 & 7.7 & 1.0 \\
\hline IPI00134549 & Lysosome-associated membrane glycoprotein 2 & Transmembrane & 8 & 9 & 0 & 17 & 100.0 & 100.0 & 0.9 \\
\hline IPI00121430 & Collagen alpha $1(\mathrm{XII})$ chain & Secreted & 11 & 14 & 0 & 25 & 100.0 & 100.0 & 0.8 \\
\hline IPI00122272 & Extracellular matrix protein 1 & Secreted & 11 & 14 & 0 & 25 & 100.0 & 100.0 & 0.8 \\
\hline IPI00227969 & Integrin alpha-6 & Transmembrane & 6 & 8 & 1 & 15 & 6.0 & 8.0 & 0.8 \\
\hline IPI00134652 & Type VII collagen & Secreted & 5 & 7 & 0 & 12 & 100.0 & 100.0 & 0.7 \\
\hline IPI00114256 & Synaptophysin-like protein & Transmembrane & 10 & 14 & 3 & 27 & 3.3 & 4.7 & 0.7 \\
\hline IPI00110810 & Prostate stem cell antigen & Secreted & 9 & 13 & 0 & 22 & 100.0 & 100.0 & 0.7 \\
\hline IPI00467180 & Translocon-associated protein beta subunit & Transmembrane & 15 & 22 & 0 & 37 & 100.0 & 100.0 & 0.7 \\
\hline IPI00133172 & Serpin B11 & Intracellular & 2 & 3 & 0 & 5 & 100.0 & 100.0 & 0.7 \\
\hline IPI00111013 & Cathepsin D & Secreted & 19 & 30 & 0 & 49 & 100.0 & 100.0 & 0.6 \\
\hline IPI00117093 & Laminin beta- 3 chain & Cell Surface & 3 & 6 & 0 & 9 & 100.0 & 100.0 & 0.5 \\
\hline IPI00130342 & Lymphocyte antigen 6 complex locus G6C protein & Secreted & 2 & 4 & 0 & 6 & 100.0 & 100.0 & 0.5 \\
\hline IPI00125293 & Eosinophil cationic protein 1 & Secreted & 1 & 2 & 0 & 3 & 100.0 & 100.0 & 0.5 \\
\hline IPI00320204 & RIKEN cDNA 2210023G05 & Secreted & 1 & 2 & 0 & 3 & 100.0 & 100.0 & 0.5 \\
\hline IPI00468097 & 340-kDa protein & Secreted & 4 & 8 & 1 & 13 & 4.0 & 8.0 & 0.5 \\
\hline IPI00113853 & Desmocollin-3 & Transmembrane & 2 & 6 & 0 & 8 & 100.0 & 100.0 & 0.3 \\
\hline
\end{tabular}


Table 2 (continued)

\begin{tabular}{|c|c|c|c|c|c|c|c|c|c|}
\hline IPI & Protein name & Protein location & $\mathrm{Ca}$ & $\mathrm{Pa}$ & $\mathrm{Nr}$ & Total & $\mathrm{Ca} / \mathrm{Nr}$ & $\mathrm{Pa} / \mathrm{Nr}$ & $\mathrm{Ca} / \mathrm{Pa}$ \\
\hline IPI00319814 & Suprabasal-specific protein suprabasin & Secreted & 3 & 10 & 0 & 13 & 100.0 & 100.0 & 0.3 \\
\hline IPI00115854 & TROP2 protein & Transmembrane & 1 & 4 & 0 & 5 & 100.0 & 100.0 & 0.3 \\
\hline IPI00127933 & Androgen binding protein alpha & Secreted & 1 & 4 & 0 & 5 & 100.0 & 100.0 & 0.3 \\
\hline IPI00130249 & GPI-anchored metastasis-associated protein homolog & Secreted & 10 & 60 & 0 & 70 & 100.0 & 100.0 & 0.2 \\
\hline IPI00111014 & Elongation of very long chain fatty acids protein 4 & Transmembrane & 3 & 20 & 2 & 25 & 1.5 & 10.0 & 0.2 \\
\hline IPI00129243 & Gamma-glutamyl hydrolase & Secreted & 1 & 8 & 0 & 9 & 100.0 & 100.0 & 0.1 \\
\hline IPI00338790 & Glandular kallikrein KLK13 & Cell Surface & 0 & 6 & 0 & 6 & 0.0 & 100.0 & 0.0 \\
\hline IPI00111115 & $\begin{array}{l}\text { Similar to METASTASIS-ASSOCIATED GPI- } \\
\text { ANCHORED PROTEIN }\end{array}$ & Secreted & 0 & 4 & 0 & 4 & 0.0 & 100.0 & 0.0 \\
\hline IPI00473830 & Biliary glycoprotein & Transmembrane & 0 & 4 & 0 & 4 & 0.0 & 100.0 & 0.0 \\
\hline IPI00153548 & Hypothetical protein & Transmembrane & 0 & 3 & 0 & 3 & 0.0 & 100.0 & 0.0 \\
\hline
\end{tabular}

$\mathrm{Ca} / \mathrm{Nr}$ : Ratio of spectral count of carcinomas to normal tissue, Pa/Nr: Ratio of spectral count of papillomas to normal tissue, Ca/Pa: Ratio of spectral count of carcinomas to papillomas

\section{Detected Tissue-Derived Proteins in Plasma}

Since the plasma proteome is dominated by several highly abundant proteins, proteins released from specific tissues would normally be present at low abundance in plasma, and their detection might be obscured by the highly abundant plasma proteins. To detect tumor-specific proteins in plasma, we used isotopic labeling to detect the isotopic peaks that consisted of the tissue-derived proteins from both plasma and tissues.

The glycopeptides from four carcinomas were labeled with $\mathrm{d} 4{ }^{13} \mathrm{C} 4$-succinic anhydride. The glycopeptides from plasma of the four mice before and after cancer development were labeled with $\mathrm{d} 0{ }^{13} \mathrm{C} 0$ and $\mathrm{d} 4{ }^{13} \mathrm{C} 0$-succinic anhydride, respectively. To monitor the labeling efficiency, we spiked the same amount of standard peptide from angiotensin $(0.1 \mu \mathrm{g})$ in the glycopeptides isolated from carcinomas and plasma samples as labeling control. Then, all the labeled peptides were combined for MS analysis. The mixture was separated by 2D Nano-LC then analyzed by MALDI-TOF/TOF. Free angiotensin (ms 1296.68) was not observed after labeling. Instead, 100, 104, and 108 Da shifted from 1296.68 were observed in equal amounts in the mixed samples. This indicates the efficient and quantitative isotopic labeling using succinic anhydride.

The mixed glycopeptides from carcinomas and plasma samples contained both skin-cancer-related peptides and peptides from plasma. In order to detect glycopeptides associated with skin cancer in plasma, we focused our analysis on glycopeptides previously identified as cancerassociated glycoproteins from skin tumors in the mixture (Table 2) and avoided the analysis of plasma proteins. To achieve this goal, the peptide peaks that contained masses from glycopeptides specifically identified from carcinomas and their isotopic pairs from plasma were selected for MS/ MS analysis.
Two types of paired patterns were observed. One was that the intensity of $\mathrm{d} 4{ }^{13} \mathrm{C} 4$-labeled peptides (with 8 mass unit shift for each amino group from peptides derived from cancer tissues) was much greater than $\mathrm{d} 44^{13} \mathrm{C} 0$-labeled peptide (with 4 mass unit shift for each amino group from peptides derived from plasma of cancer-bearing mice), and intensity of $\mathrm{d} 0{ }^{13} \mathrm{C} 0$-labeled peptide (with 0 mass unit shift for each amino group from peptides derived from plasma before carcinogen induction) was lower than that of peptides from plasma of cancer-bearing mice. This pattern indicated that the peptide was from tumor-specific protein and detectable in cancer plasma at low intensity. The other pattern was that similar or lower intensity of peptides from cancer tissues than in plasma, and peptides with this pattern were derived from plasma proteins.

Tumor-associated glycopeptides could be detected in plasma. Tenascin-C was identified in carcinomas with 133 spectra, and it was also identified in benign papillomas with 29 spectra. However, none of these glycopeptides were identified in normal tissue (Table 2). In plasma, the labeled peptide peak of Tenascin-C from cancer was found with its paired peak from cancer plasma (Fig. 2A), which indicated that it was also detected in plasma after cancer development, but not in control plasma before the carcinogen treatment. Another skin tumor-specific glycoprotein, Arylsulfatase B, was also detected in plasma successfully in a similar way (Fig. 2b). These data indicated that extracellular proteins associated with tumor development were identifiable in plasma from tumor-bearing mice using glycopeptide capture, isotopic labeling, and mass spectrometry.

One of the advantages of using this tissue-targeted approach is that tumor-associated proteins can be identified in plasma even if they are present in very low abundance. The peptides from cancer tissue are likely to be at higher abundance compared to the same peptides in plasma. This allowed us to determine their masses and peptide sequences in the mixture 
Fig. 2 Detection of tumor-specific proteins in plasma. a The detected paired peaks of succinic-anhydride-labeled Tenascin-C and MS/MS spectrum of Tenascin-C. b The paired peak of succinic-anhydridelabeled Arylsulfatase B and MS/MS of Arylsulfatase B. c The paired peak of succinic-anhydride-labeled Ig gamma-3 chain $\mathrm{C}$ region showed different peak pattern from Tenascin-C and MS/MS of Ig gamma-3 chain $\mathrm{C}$ region. $\mathrm{NrP}$ mouse plasma without carcinogen treatment, $\mathrm{CaP}$ mouse plasma from cancer-bearing mice after carcinogen treatment, $M T$ mouse cancer tissues

using isotopic peaks from tumors. Using this information, tumor-derived peptides in plasma can be identified, while they are not identifiable by data-dependent MS/MS acquisition and database searches. Both Tenascin-C and Arylsulfatase B are low abundant proteins. They were not identified in plasma before cancer development, and their detection in plasma was associated with cancer development.

Proteins from plasma can also be detected in tissues and plasma as isotopic pairs due to vascularization of the tissue. If a glycopeptide detected in both cancer tissues and plasma was derived from plasma, the peptide peak showed similar or lower intensity in cancer tissues than that in plasma. An example of this was the identification and quantification of glycoprotein, Ig gamma- 3 chain $\mathrm{C}$ region, in tissue and plasma. However, its paired peptide peaks were found in a different pattern from that observed with Tenascin-C (Fig. 2c). The intensities of $\mathrm{d} 0{ }^{13} \mathrm{C} 0$ and $\mathrm{d} 4{ }^{13} \mathrm{C} 0$-labeled peptides from plasma before and after tumor induction were much higher than that from $\mathrm{d} 4{ }^{13} \mathrm{C} 4$ labeled peptides from tumors. This indicated that this peptide was from a plasma-derived protein, and Ig gamma-3 could be detected from tissue due to the blood contamination in the tumor.

The methodology of targeted detection of tumor proteins using glycopeptide capture, isotopic labeling, and mass spectrometry is based on the analysis of N-linked glycopeptides to study extracellular proteins from tumors and plasma. It has been shown to increase the detectability of tumor proteins by focusing on the same subset of glycopeptides in both tumors and plasma [13]. The tumorassociated glycopeptides could be detected in plasma on account of the several advantages of our methodologies. First, the glycopeptide capture method dramatically reduces the sample complexity. Non-glycoproteins and non-glycopeptides from glycoproteins were removed from the pool of samples. For example, albumin, the most abundant serum protein, was automatically transparent to this method since it does not contain N-linked glycosylation. Second, the glycopeptide isolation method could be used to enrich extracellular proteins due to the fact that most extracellular proteins are glycosylated and likely to enter the bloodstream. Third, we used an isotopic labeling method to facilitate the detection of tumor proteins within complex plasma by identifying paired peptide peaks from tumor tissues and plasma. However, the method described a
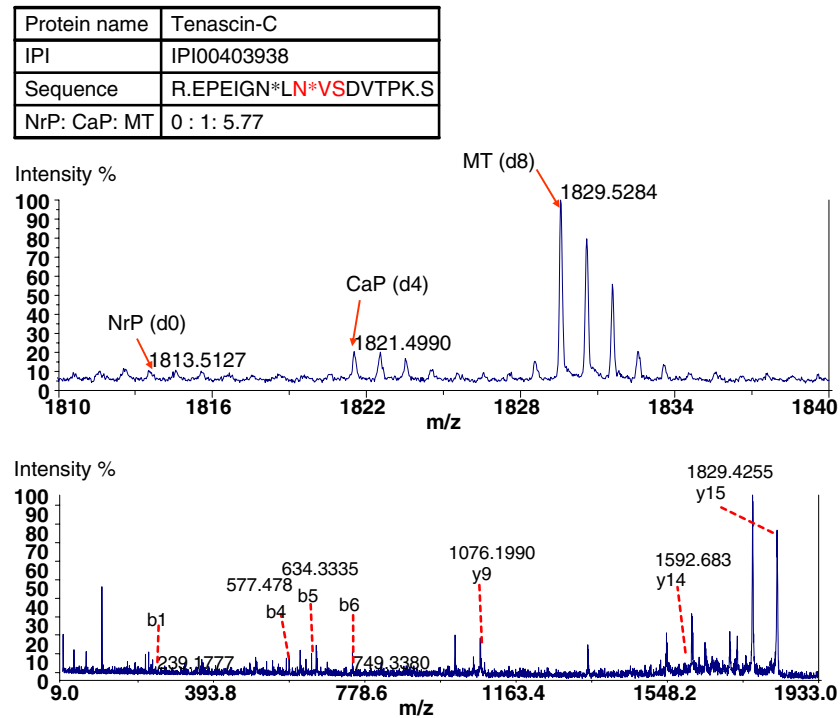

b
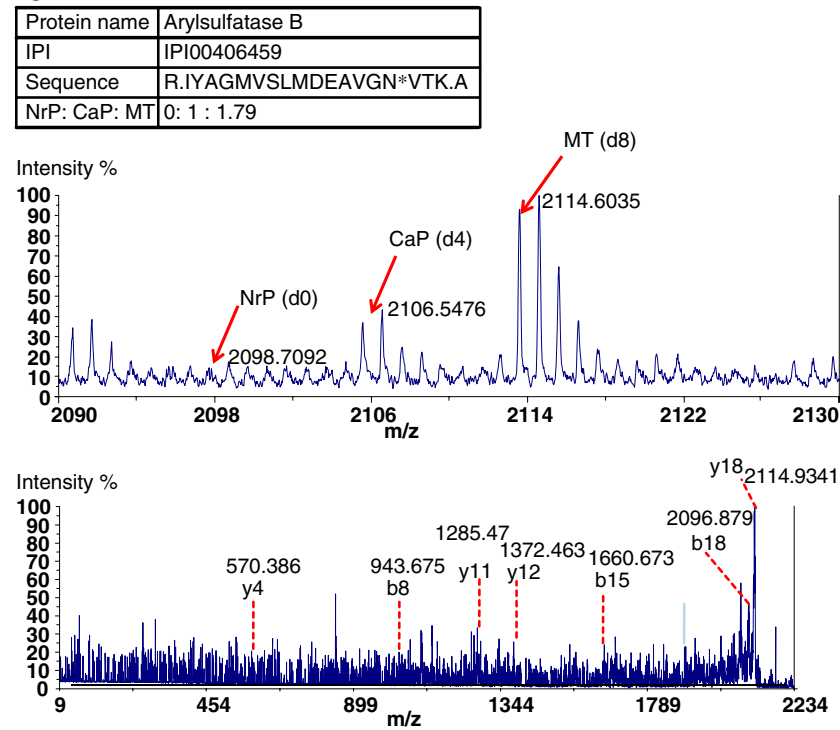

C
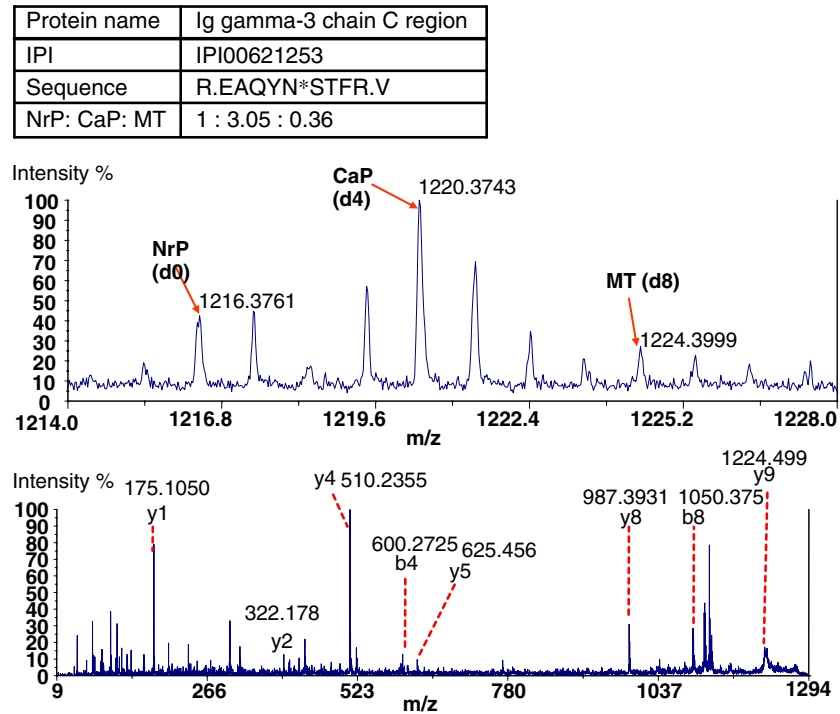
here is only for proteins that contain N-linked glycosylation. For proteins that do not contain N-linked glycosylation, this method will miss the detection of those proteins.

These results show that our strategy for detection of tumor-specific proteins in plasma is specific and sensitive for low abundant tumor-associated proteins. Different from the previous report of identification of prostate cancer-derived proteins in serum using xenograft-bearing mice [30], our study is more focused on tumor-associated extracellular proteins that are likely to be used in early detection.

\section{Conclusions}

In this study, we described a platform for quantitative detection of tumor-specific extracellular proteins derived from tumors and plasma. The fact that tumor-specific proteins were detectable in plasma from tumor-bearing mice indicates that cancer-specific markers could be detected in plasma using targeted approaches, and these proteins could be serum tumor marker candidates [7]. Once such candidate proteins are identified, the homologues of the proteins can be verified in human sera using the targeted approach. Enzyme-linked immunosorbent assays can be developed using a pair of antibodies. However, if antibodies against the candidate proteins are not available, mass-spectrometry-based methods can be applied to detect candidate proteins in plasma. One approach is referred to as MRM [17-19]. In another approach, called stable isotope standards and capture by anti-peptide antibodies, a specific peptide from sample and the synthetic heavy isotope-labeled peptide of the candidate protein are captured by peptide antibody. The mass spectrometer is then used to detect and quantify the specific peptide with known precursor mass and fragmentation ions [31].

Acknowledgment This work was supported with federal funds from the National Cancer Institute, National Institutes of Health, by Grants R21-CA-114852. We gratefully acknowledge the support from the Mass Spectrometry Facility at the Johns Hopkins University and the support of Trans-Proteomic Pipeline (TPP) Software tools available from the Aebersold Group at the Institute for Systems Biology.

\section{References}

1. Etzioni R, et al. The case for early detection. Nat Rev Cancer. 2003;3:243-52.

2. Pieper R, et al. Multi-component immunoaffinity subtraction chromatography: an innovative step towards a comprehensive survey of the human plasma proteome. Proteomics. 2003;3:422-32.

3. Adkins JN, et al. Toward a human blood serum proteome: analysis by multidimensional separation coupled with mass spectrometry. Mol Cell Proteomics. 2002;1:947-55.

4. Tirumalai RS, et al. Characterization of the low molecular weight human serum proteome. Mol Cell Proteomics. 2003;2:1096-103.
5. Shen Y, et al. Ultra-high-efficiency strong cation exchange LC/ RPLC/MS/MS for high dynamic range characterization of the human plasma proteome. Anal Chem. 2004;76:1134-44.

6. Coombes KR. Analysis of mass spectrometry profiles of the serum proteome. Clin Chem. 2005;51:1-2.

7. Zhang H, Chan DW. Cancer biomarker discovery in plasma using a tissue-targeted proteomic approach. Cancer Epidemiol Biomarkers Prev. 2007;16:1915-7.

8. Zhang H, Li XJ, Martin DB, Aebersold R. Identification and quantification of N-linked glycoproteins using hydrazide chemistry, stable isotope labeling and mass spectrometry. Nat Biotechnol. 2003;21:660-6.

9. Tian Y, Zhou Y, Elliott S, Aebersold R, Zhang H. Solid-phase extraction of N-linked glycopeptides. Nat Protoc. 2007;2: 334-9.

10. Zhou Y, Aebersold R, Zhang H. Isolation of N-linked glycopeptides from plasma. Anal Chem. 2007;79:5826-37.

11. Roth J. Protein N-glycosylation along the secretory pathway: relationship to organelle topography and function, protein quality control, and cell interactions. Chem Rev. 2002;102: 285-303.

12. Zhang $\mathrm{H}$, et al. High throughput quantitative analysis of serum proteins using glycopeptide capture and liquid chromatography mass spectrometry. Mol Cell Proteomics. 2005;4:144-55.

13. Zhang $\mathrm{H}$, et al. Mass spectrometric detection of tissue proteins in plasma. Mol Cell Proteomics. 2007;6:64-71.

14. Schulz BL, Laroy W, Callewaert N. Clinical laboratory testing in human medicine based on the detection of glycoconjugates. Curr Mol Med. 2007;7:397-416.

15. Pan S, et al. High throughput proteome screening for biomarker detection. Mol Cell Proteomics. 2005;4:182-90.

16. Zhang H, Yan W, Aebersold R. Chemical probes and tandem mass spectrometry: a strategy for the quantitative analysis of proteomes and subproteomes. Curr Opin Chem Biol. 2004;8:66-75.

17. Keshishian H, Addona T, Burgess M, Kuhn E, Carr SA. Quantitative, multiplexed assays for low abundance proteins in plasma by targeted mass spectrometry and stable isotope dilution. Mol Cell Proteomics. 2007;6:2212-29.

18. Stahl-Zeng J, et al. High sensitivity detection of plasma proteins by multiple reaction monitoring of $\mathrm{N}$-glycosites. Mol Cell Proteomics. 2007;6:1809-17.

19. Anderson L, Hunter CL. Quantitative mass spectrometric multiple reaction monitoring assays for major plasma proteins. Mol Cell Proteomics. 2006;5:573-88.

20. Kemp CJ. Multistep skin cancer in mice as a model to study the evolution of cancer cells. Semin Cancer Biol. 2005;15:460-73.

21. Eng J, McCormack AL, Yates JR 3rd. An approach to correlate tandem mass spectral data of peptides with amino acid sequences in a protein database. J Am Soc Mass Spectrom. 1994;5:976-89.

22. Han DK, Eng J, Zhou H, Aebersold R. Quantitative profiling of differentiation-induced microsomal proteins using isotope-coded affinity tags and mass spectrometry. Nat Biotechnol. 2001; 19:946-51.

23. Keller A, Nesvizhskii AI, Kolker E, Aebersold R. Empirical statistical model to estimate the accuracy of peptide identifications made by MS/MS and database search. Anal Chem. 2002;74:5383-92.

24. Whiteaker JR, et al. Integrated pipeline for mass spectrometrybased discovery and confirmation of biomarkers demonstrated in a mouse model of breast cancer. J Proteome Res. 2007;6: 3962-75.

25. Zou Z, et al. Synthesis and evaluation of superparamagnetic silica particles for extraction of glycopeptides in the microtiter plate format. Anal Chem. 2008;80:1228-34.

26. Liu H, Sadygov RG, Yates JR 3rd. A model for random sampling and estimation of relative protein abundance in shotgun proteomics. Anal Chem. 2004;76:4193-201. 
27. Jones FS, Jones PL. The tenascin family of ECM glycoproteins: structure, function, and regulation during embryonic development and tissue remodeling. Dev Dyn. 2000;218:235-59.

28. Orend G. Potential oncogenic action of tenascin-C in tumorigenesis. Int J Biochem Cell Biol. 2005;37:1066-83.

29. Ghosh D. Human sulfatases: a structural perspective to catalysis. Cell Mol Life Sci. 2007;64:2013-22.
30. van den Bemd GJ, et al. Mass spectrometric identification of human prostate cancer-derived proteins in serum of xenograftbearing mice. Mol Cell Proteomics. 2006;5:1830-39.

31. Anderson NL, et al. Mass spectrometric quantitation of peptides and proteins using stable isotope standards and capture by anti-peptide antibodies (SISCAPA). J Proteome Res. 2004; $3: 235-44$. 